\title{
APPLICATIONS OF FACTORIZATION EMBEDDINGS FOR LÉVY PROCESSES
}

\author{
A. B. DIEKER, ${ }^{*}$ CWI Amsterdam and University of Twente
}

\begin{abstract}
We give three applications of the Pecherskii-Rogozin-Spitzer identity for Lévy processes. First, we find the joint distribution of the supremum and the epoch at which it is 'attained' if a Lévy process has phase-type upward jumps. We also find the characteristics of the ladder process. Second, we establish general properties of perturbed risk models, and obtain explicit fluctuation identities in the case that the Lévy process is spectrally positive. Third, we study the tail asymptotics for the supremum of a Lévy process under different assumptions on the tail of the Lévy measure.
\end{abstract}

Keywords: First factorization identity; Lévy process; perturbed risk model; phase-type jump; ruin probability

2000 Mathematics Subject Classification: Primary 60J30

Secondary 60G70; 91B30

\section{Introduction}

Fluctuation theory analyzes quantities related to the extrema of a stochastic process. Examples include the distribution of the supremum or infimum, the last (or first) time that the process attains its extremum, first passage times, overshoots, and undershoots. The study of these distributions is often motivated by applications in queueing theory, mathematical finance, or insurance mathematics.

Of particular interest are the fluctuations of a Lévy process $Z$. Such a process has stationary and independent increments, and is defined on the probability space of càdlàg functions (i.e. those that are continuous from the right with left limits) with the Borel $\sigma$-field generated by the usual Skorokhod topology. The characteristic function of $Z_{t}$ necessarily has the form $\mathrm{Ee}^{\mathrm{i} \beta Z_{t}}=\mathrm{e}^{-t \Psi_{Z}(\beta)}, \beta \in \mathbb{R}$, where

$$
\Psi_{Z}(\beta)=\frac{1}{2} \sigma_{Z}^{2} \beta^{2}+\mathrm{i} c_{Z} \beta+\int_{\mathbb{R}}\left(1-\mathrm{e}^{\mathrm{i} \beta z}+\mathrm{i} \beta z 1(|z| \leq 1)\right) \Pi_{Z}(\mathrm{~d} z),
$$

for some $\sigma_{Z} \geq 0$ and $c_{Z} \in \mathbb{R}$ and a so-called Lévy measure, $\Pi_{Z}$, on $\mathbb{R} \backslash\{0\}$ satisfying $\int\left(1 \wedge|z|^{2}\right) \Pi_{Z}(\mathrm{~d} z)<\infty$. In particular, $Z_{0}=0$, and $Z$ is called a compound Poisson process if $c_{Z}=\sigma_{Z}=0$ and $\Pi_{Z}(\mathbb{R})<\infty$.

It is the objective of this paper to show how one specific technique, the so-called factorization embedding, can be successfully used in a variety of applied probability models related to the fluctuations of a Lévy process $Z$. We consider three applications of this embedding approach in the present work. All three applications have been studied in the literature using other techniques; in order to illustrate the power of the method, we show that factorization embeddings provide a simple way to extend the known results.

Received 4 August 2005; revision received 18 April 2006.

* Current address: University College Cork, BCRI, 17 South Bank, Crosses Green, Cork, Ireland.

Email address: t.dieker@proba.ucc.ie 

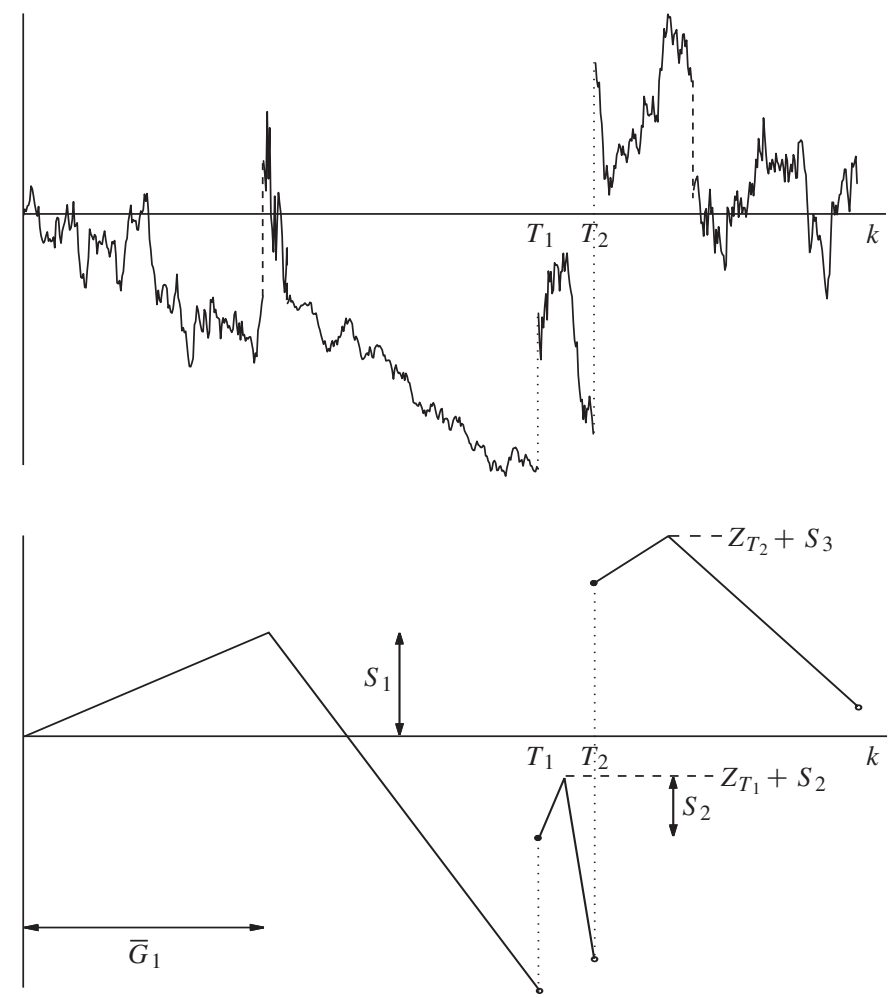

FIGURE 1: A realization of the killed Lévy process $Z=X+Y$ and the corresponding embedded (piecewiselinear) process. Jumps of $Y$ are dotted and jumps of $X$ are dashed.

\subsection{Factorization embeddings}

The central object of this paper is a Lévy process $Z$. A factorization embedding is a process that is determined by considering $Z$ at (countably many) specific epochs. Before the embedding can be defined, $Z$ has to be written as the sum of an arbitrary one-dimensional Lévy process, $X$, and a compound Poisson process, $Y$, with intensity, $\lambda$, independent of $X$. Note that any discontinuous Lévy process $Z$ can be written in this form; this paper does not focus on continuous Lévy processes (i.e. Brownian motions with drift), since their fluctuation theory is well established. The representation $Z=X+Y$ need not be unique; for instance, there is a continuum of such representations if the Lévy measure has a nonvanishing absolutely continuous part.

Before explaining the idea behind the embedding that we study, we first introduce some notation. Write $T_{1}, T_{2}, \ldots$ for the jump epochs of $Y$, and set $T_{0}=0$. Define the quantities $\bar{G}_{i}$ and $S_{i}$, for $i \geq 1$, as follows. By $Z_{T_{i-1}}+S_{i}$ we represent the value of the supremum within $\left[T_{i-1}, T_{i}\right)$, and $T_{i-1}+\bar{G}_{i}$ is the last epoch in this interval such that the value of $Z$ at $T_{i-1}+\bar{G}_{i}$ or $\left(T_{i-1}+\bar{G}_{i}\right)-$ is $Z_{T_{i-1}}+S_{i}$. Although formally incorrect, we say in the remainder of the paper that the supremum of $Z$ over $\left[T_{i-1}, T_{i}\right.$ ) is attained at $T_{i-1}+\bar{G}_{i}$, with value $Z_{T_{i-1}}+S_{i}$.

In the upper plot of Figure 1, a realization of $Z$ is given. The jumps of $Y$ are dotted and those of $X$ are dashed. The process $Z$ is killed at an exponentially distributed random time 
$k$, independent of $Z$, say with parameter $q \geq 0$ ( $q=0$ corresponds to no killing). The lower plot in Figure 1 is obtained from the upper one by replacing the trajectory of $Z$ between $T_{i-1}$ and $T_{i}$ by a piecewise-straight line consisting of two pieces: one from $\left(T_{i-1}, Z_{T_{i-1}}\right)$ to $\left(T_{i-1}+\bar{G}_{i}, Z_{T_{i-1}}+S_{i}\right)$, and one from the latter point to $\left(T_{i}, Z_{T_{i}-}\right)$. These two pieces together have the shape of a 'hat'. The crucial observation is that the embedded piecewise-linear process still contains all information on key fluctuation quantities like the global supremum of $Z$ and the epoch at which it is attained for the last time.

The piecewise-linear process has several other useful properties. First, by the Markov property, the 'hats' are mutually independent given their starting point. Moreover, obviously, the jumps of $Y$ are independent of the 'hats'. More strikingly, the increasing and decreasing pieces of each 'hat' are also independent: indeed, $\left(T_{i}-T_{i-1}, Z_{T_{i}-}-Z_{T_{i-1}}\right)=\left(\bar{G}_{i}, S_{i}\right)+$ $\left(T_{i}-T_{i-1}-\bar{G}_{i}, Z_{T_{i}-}-Z_{T_{i-1}}-S_{i}\right)$, where the two latter vectors are independent; cf. the Pecherskii-Rogozin-Spitzer factorization for Lévy processes (Proposition 1, below). This explains the name factorization embedding.

The lower plot in Figure 1 can be generated without knowledge of the trajectory of $Z$. Indeed, since $\left\{T_{i}: i \geq 1\right\}$ is a Poisson point process with intensity $\lambda$ and killing rate $q$, it is equivalent (in law) to the first $N$ points of a Poisson point process with intensity $\lambda+q$, where $N$ is geometrically distributed on $\mathbb{Z}_{+}$with parameter $\lambda /(\lambda+q)$ (independent of the point process).

It is not a new idea to consider an embedded process to study fluctuations of Lévy processes. A classical example with $q=0$ is when $X$ has negative drift $(X(t)=c t$ for some $c<0)$ and $Y$ only has positive jumps. We then have $\bar{G}_{i}=S_{i}=0$ for every $i$, and $\left(\bar{G}_{i}, S_{i}\right)+\left(T_{i}-\right.$ $\left.T_{i-1}-\bar{G}_{i}, Z_{T_{i}-}-Z_{T_{i-1}}-S_{i}\right)$ is distributed as $\left(e_{\lambda}, c e_{\lambda}\right)$, where $e_{\lambda}$ denotes an exponentially distributed random variable with parameter $\lambda$. Therefore, a random walk can be studied in order to analyze the fluctuations of $Z$. To the author's knowledge, nontrivial factorization embeddings have only been used to obtain results in the space domain. We mention the work of Kennedy [23] and Asmussen [2], who studied certain Markov additive processes, and the work of Mordecki [28], who studied the supremum of a Lévy process with phase-type upward jumps and general downward jumps. Recently, a slightly different form of this embedding has been used by Doney [16] to derive stochastic bounds on the Lévy processes $Z$. He defined $X$ and $Y$ such that the supports of $\Pi_{X}$ and $\Pi_{Y}$ are disjoint, and noticed that $\left\{Z_{T_{i-1}}+S_{i}\right\}$ is a random walk with a random starting point, meaning that it suffices to establish stochastic bounds on the starting point. Doney then used these to analyze the asymptotic behavior of Lévy processes that converge to $\infty$ in probability. As an aside, we remark that the factorization embedding is different from the embedding that has been used in [5] and [30], where jumps are absorbed by some random environment.

\subsection{Outline and contribution of the paper: three applications}

We now describe how this paper is organized, thereby introducing the three problems that are to be studied using factorization embeddings. All the results in this paper are new, the only exceptions being Proposition 1, Theorem 5, and the first claim of Theorem 7.

Section 2 is a preliminary section, in which background is given and the above idea is used to express fluctuation quantities of $Z$ in terms of those of $X$.

Section 3 uses the above embedding idea to study the case where $Z$ has phase-type upward jumps and general downward jumps. Then the Laplace exponent, $\kappa_{Z}$, of the bivariate ladder process can be given; this is a quantity that lies at the heart of fluctuation theory for Lévy processes; see Chapter VI of [7]. In particular, we give the joint law of the supremum and the epoch at which it is 'attained', generalizing Mordecki's [28] results. 
Section 4 studies perturbed risk models, a generalization of classical risk models that has drawn much attention in the literature. We prove a general Pollaczek-Khinchin formula in this framework, but explicit results can only be obtained under further assumptions. Therefore, we impose spectral positivity of the Lévy process underlying the risk model and extend the recent results of Huzak et al. [20] in the following way. While [20] focuses on quantities related to so-called modified ladder heights, we obtain joint distributions related to both modified ladder epochs and modified ladder heights. In particular, we obtain the (transform of the) distribution of the first modified ladder epoch.

Section 5 studies the tail of the supremum of $Z$ under three different assumptions on the Lévy measure. We reproduce known results in the Cramér case and the subexponential case, but in the latter case also give a local variant which is new. Our results for the intermediate case are also new, and complement recent work of Klüppelberg et al. [24].

The above selection of three applications may seem fairly random. This is indeed the case in the sense that the use of factorization embeddings is not limited to these three applications. For instance, Dębicki et al. [14] applied similar ideas to investigate Lévy-driven fluid queue networks.

After finishing this paper, we learned about the work of Pistorius [30]. There is some overlap between his work and our Section 3 in the special case $K=1$ (see below). In [30], the Laplace exponent, $\kappa_{Z}$, of the ladder process was characterized in terms of the solutions to the equation $\Psi_{Z}(\beta)=q$. Our approach is different, since we express $\kappa_{Z}(q, \beta)$ in terms of a vector $\boldsymbol{\alpha}_{+}^{q}$, for which we give an efficient algorithm.

\section{On factorization identities}

In this section, we consider the process $Z=X+Y$, where $Y$ is a compound Poisson process and $X$ is a general Lévy process, independent of $Y$. After giving some background in Subsection 2.1, we study the supremum and infimum of $Z$ and the epoch at which they are attained for the first (or last) time in Subsection 2.2. We express their joint distribution in terms of the corresponding distribution of $X$. Moreover, the characteristics of the bivariate ladder process of $Z$ are expressed in terms of those of $X$.

\subsection{Background}

We start with some notation. Given a Lévy process $X$, we define

$$
\begin{aligned}
\bar{X}_{t} & =\sup \left\{X_{s}: 0 \leq s \leq t\right\}, & & \underline{X}_{t}=\inf \left\{X_{s}: 0 \leq s \leq t\right\}, \\
\bar{F}_{t}^{X} & =\inf \left\{s<t: X_{s}=\bar{X}_{t} \text { or } X_{s-}=\bar{X}_{t}\right\}, & & \bar{G}_{t}^{X}=\sup \left\{s<t: X_{s}=\bar{X}_{t} \text { or } X_{s-}=\bar{X}_{t}\right\}, \\
\underline{F}_{t}^{X} & =\inf \left\{s<t: X_{s}=\underline{X}_{t} \text { or } X_{s-}=\underline{X}_{t}\right\}, & & \underline{G}_{t}^{X}=\sup \left\{s<t: X_{s}=\underline{X}_{t} \text { or } X_{s-}=\underline{X}_{t}\right\} .
\end{aligned}
$$

The following identity, referred to as the Pecherskii-Rogozin-Spitzer (PRS) identity in the remainder of the paper, is key to the results of the paper. Here and throughout, $e_{q}$ denotes an exponentially distributed random variable with parameter $q$, independent of $X$ and $Y$. For an account of the history of this identity, we refer the reader to [7, p. 185].

Proposition 1. (Pecherskii-Rogozin-Spitzer.) For $\alpha \geq 0, \beta \in \mathbb{R}$, and $q>0$, we have

$$
\begin{aligned}
\mathrm{E} \exp \left(-\alpha e_{q}+\mathrm{i} \beta X_{e_{q}}\right) & =\mathrm{E} \exp \left(-\alpha \bar{G}_{e_{q}}^{X}+\mathrm{i} \beta \bar{X}_{e_{q}}\right) \mathrm{E} \exp \left(-\alpha \underline{F}_{e_{q}}^{X}+\mathrm{i} \beta \underline{X}_{e_{q}}\right) \\
& =\mathrm{E} \exp \left(-\alpha \bar{F}_{e_{q}}^{X}+\mathrm{i} \beta \bar{X}_{e_{q}}\right) \mathrm{E} \exp \left(-\alpha \underline{G}_{e_{q}}^{X}+\mathrm{i} \beta \underline{X}_{e_{q}}\right) .
\end{aligned}
$$


The second equality follows from the first by considering the dual process $\hat{X}=-X$. The PRS identity is sometimes referred to as the first factorization identity. It can be viewed as a decomposition of $\left(e_{q}, X{ }_{e_{q}}\right)$ into the sum of two independent vectors, since $\left(\underline{F}_{e_{q}}^{X}, \underline{X}_{e_{q}}\right)$ is distributed as is $\left(e_{q}-\bar{G}_{e_{q}}^{X}, X_{e_{q}}-\bar{X}_{e_{q}}\right)$.

In order to relate the PRS factors of $Z$ and $X$, we need an auxiliary random walk. We write $\lambda \in(0, \infty)$ for the intensity of $Y$, and $\xi$ for its generic jump. For a fixed $q>0$, let $\left\{S_{n}^{q}\right\}$ be a random walk with step size distribution $\xi+X_{e_{\lambda+q}}$, where the two summands are independent. For this random walk, we define the first strict ascending and descending ladder epochs as, respectively,

$$
\tau_{\mathrm{s}+}^{q}=\inf \left\{n \geq 1: S_{n}^{q}>0\right\}, \quad \tau_{\mathrm{s}-}^{q}=\inf \left\{n \geq 1: S_{n}^{q}<0\right\},
$$

and we define $\tau_{\mathrm{w}+}^{q}$ and $\tau_{\mathrm{w}-}^{q}$ similarly, with weak inequalities. We write $H_{\mathrm{w} \pm}^{q}$ and $H_{\mathrm{s} \pm}^{q}$ for the ladder heights $S_{\tau_{\mathrm{w} \pm}^{q}}^{q}$ and $S_{\tau_{\mathrm{s} \pm}^{q}}^{q}$, respectively.

When integrating with respect to defective distributions, we only carry out the integration over the set where the random variables are both finite and well defined. For instance, we write $\mathrm{E} \exp \left(-\beta H_{\mathrm{s}+}^{q}\right) \rho^{\tau_{\mathrm{s}+}^{q}}$ for $\mathrm{E}\left[\exp \left(-\beta H_{\mathrm{s}+}^{q}\right) \rho^{\tau_{\mathrm{s}+}^{q}} ; \tau_{\mathrm{s}+}^{q}<\infty\right]$ in the remainder of the paper, unless indicated otherwise.

\subsection{The PRS factorization and ladder characteristics}

The main result of this section, which we now formulate, relates the PRS factors of $Z$ and $X$. When a specific structure is imposed on $X$ and $Y$, both factors can be computed; see Section 3 . Intuitively, a PRS factor of $Z$ is the product of a PRS factor of $X$ and a random-walk PRS factor. The main complication is that the random walk is converted to a continuous-time process by 'stretching' time, but that this stretching is not done independently of the step size.

Theorem 1. Suppose that $Z$ can be written as $Z=X+Y$ for independent processes $X$ and $Y$, where $Y$ is a compound Poisson process. For every $\alpha, \beta \geq 0$ and $q>0$, we have

$$
\begin{aligned}
& \mathrm{E} \exp \left(-\alpha \bar{G}_{e_{q}}^{Z}-\beta \bar{Z}_{e_{q}}\right) \\
& \quad=\operatorname{E} \exp \left(-\alpha \bar{G}_{e_{\lambda+q}}^{X}-\beta \bar{X}_{e_{\lambda+q}}\right) \frac{1-\mathrm{E}(\lambda /(\lambda+q))^{\tau_{\mathrm{w}+}^{q}}}{1-\mathrm{E} \exp \left(-\beta H_{\mathrm{w}+}^{q+\alpha}\right)(\lambda /(\lambda+q+\alpha))^{\tau_{\mathrm{w}+}^{q+\alpha}}}, \\
& \mathrm{E} \exp \left(-\alpha \bar{F}_{e_{q}}^{Z}-\beta \bar{Z}_{e_{q}}\right) \\
& \quad=\operatorname{E} \exp \left(-\alpha \bar{F}_{e_{\lambda+q}}^{X}-\beta \bar{X}_{e_{\lambda+q}}\right) \frac{1-\mathrm{E}(\lambda /(\lambda+q))^{\tau_{\mathrm{s}+}^{q}}}{1-\mathrm{E} \exp \left(-\beta H_{\mathrm{s}+}^{q+\alpha}\right)(\lambda /(\lambda+q+\alpha))^{\tau_{\mathrm{s}+}^{q+\alpha}}},
\end{aligned}
$$

while $\mathrm{E} \exp \left(-\alpha \underline{F}_{e_{q}}^{Z}+\mathrm{i} \beta \underline{Z}_{e_{q}}\right)$ and $\mathrm{E} \exp \left(-\alpha \underline{G}_{e_{q}}^{Z}+\mathrm{i} \beta \underline{Z}_{e_{q}}\right)$ follow by duality.

Proof. We only prove the first equality; the argument is easily adapted to obtain the second.

The first factor is a direct consequence of the independence of the first straight line in the lower plot of Figure 1 and the other pieces; see the remarks accompanying Figure 1. Writing $w_{i}=T_{i-1}+\bar{G}_{i}-\bar{G}_{1}$ and $W_{i}=Z_{T_{i-1}+\bar{G}_{i}}-S_{1}$, for $i \geq 1$, these arguments also reveal that $\left\{W_{i}: i \geq 1\right\}$ is a random walk with the same distribution as $\left\{S_{n}^{q}: n \geq 0\right\}$, except for the killing in every step with probability $\lambda /(\lambda+q)$. Therefore, if we define the first (weak) ascending ladder epoch of this random walk as $N=\inf \left\{i \geq 1: W_{i} \geq 0\right\}$, we have

$$
\mathrm{P}(N<\infty)=\mathrm{E}\left(\frac{\lambda}{\lambda+q}\right)^{\tau_{\mathrm{w}+}^{q}} .
$$


Observe that $\left(\bar{G}_{e_{q}}^{Z}-\bar{G}_{1}, \bar{Z}_{e_{q}}-S_{1}\right)$ has the same distribution as

$$
\sum_{j=1}^{K}\left(w_{N}^{j}, W_{N}^{j}\right)
$$

where $K$ is geometrically distributed on $\mathbb{Z}_{+}$with parameter $\mathrm{P}(N<\infty)$ and $\left(w_{N}^{j}, W_{N}^{j}\right)$ are independent copies of $\left(w_{N}, W_{N}\right)$ also independent of $K$. Note that we consider the weak ladder epoch in the definition of $N$, since we are interested in $\bar{G}_{e_{q}}^{Z}$ (as opposed to $\bar{F}_{e_{q}}^{Z}$ ). This shows that

$$
\mathrm{E} \exp \left(-\alpha\left(\bar{G}_{e_{q}}^{Z}-\bar{G}_{1}\right)+\mathrm{i} \beta\left(\bar{Z}_{e_{q}}-S_{1}\right)\right)=\frac{1-\mathrm{E}(\lambda /(\lambda+q))^{\tau_{\mathrm{w}+}^{q}}}{1-\mathrm{E}(\lambda /(\lambda+q))^{N} \mathrm{e}^{-\alpha w_{N}+\mathrm{i} \beta W_{N}}},
$$

and it remains to study the denominator in more detail.

For this, we rely on Section I.1.12 of [31]. The key observation is that $\left\{\left(w_{i}, W_{i}\right)\right\}$ is a random walk in the half-plane $\mathbb{R}_{+} \times \mathbb{R}$, with step size distribution characterized by

$$
\mathrm{Ee}^{-\alpha w_{1}+\mathrm{i} \beta W_{1}}=\mathrm{E} \exp \left(-\alpha e_{\lambda+q}+\mathrm{i} \beta X_{e_{\lambda+q}}\right) \mathrm{Ee}^{\mathrm{i} \beta \xi} .
$$

Theorem 27 of [31], which is a Wiener-Hopf factorization for random walks on the half-plane, shows that, for $|z|<1, \alpha \geq 0$, and $\beta \in \mathbb{R}$, we may write

$$
1-z \mathrm{E} \exp \left(-\alpha e_{\lambda+q}+\mathrm{i} \beta X_{e_{\lambda+q}}\right) \mathrm{E} \mathrm{e}^{\mathrm{i} \beta \xi}=\left[1-\mathrm{E} z^{N} \mathrm{e}^{-\alpha w_{N}+\mathrm{i} \beta W_{N}}\right]\left[1-\mathrm{E} z^{\bar{N}} \mathrm{e}^{-\alpha w_{\bar{N}}+\mathrm{i} \beta W_{\bar{N}}}\right],
$$

where the bars refer to (strict) descending ladder variables. The actual definitions of these quantities are of minor importance to us; the crucial point is that this factorization is unique. Indeed, an alternative characterization,

$$
1-z \mathrm{E} \exp \left(-\alpha e_{\lambda+q}+\mathrm{i} \beta X_{e_{\lambda+q}}\right) \mathrm{Ee}^{\mathrm{i} \beta \xi}=1-\frac{(\lambda+q) z}{\lambda+q+\alpha} \mathrm{E} \exp \left(\mathrm{i} \beta X_{e_{\lambda+q+\alpha}}\right) \mathrm{Ee}^{\mathrm{i} \beta \xi},
$$

is obtained by conditioning on the value of $e_{\lambda+q}$, and the Wiener-Hopf factorization for random walks shows that this can be written as

$$
\left[1-\mathrm{E}\left(\frac{(\lambda+q) z}{\lambda+q+\alpha}\right)^{\tau_{\mathrm{s}+}^{q+\alpha}} \mathrm{e}^{\mathrm{i} \beta H_{\mathrm{s}+}^{q+\alpha}}\right]\left[1-\mathrm{E}\left(\frac{(\lambda+q) z}{\lambda+q+\alpha}\right)^{\tau_{\mathrm{w}-}^{q+\alpha}} \mathrm{e}^{\mathrm{i} \beta H_{\mathrm{w}-}^{q+\alpha}}\right] .
$$

This decomposition is again unique, so the claim follows by substituting $z=\lambda /(\lambda+q)$. This finishes the proof.

If $\alpha=0$ then we must have $\operatorname{Eexp}\left(-\alpha \bar{G}_{e_{q}}^{Z}-\beta \bar{Z}_{e_{q}}\right)=\operatorname{E} \exp \left(-\alpha \bar{F}_{e_{q}}^{Z}-\beta \bar{Z}_{e_{q}}\right)$, but the formulae in Theorem 1 differ in the sense of weak and strict ladder variables. This is not a contradiction, as Spitzer's identity shows that the fractions are equal for both $\tau_{\mathrm{w}+}^{q}$ and $\tau_{\mathrm{s}+}^{q}$.

Let us now verify that the formulae of Theorem 1 are in accordance with the PRS factorization of Proposition 1. Indeed, with the Wiener-Hopf factorization for random walks (1) and Theorem 1 (the transform $\operatorname{Exp}\left(-\alpha \underline{F}_{e_{q}}^{Z}+\mathrm{i} \beta \underline{Z}_{e_{q}}\right)$ is obtained by duality), we have

$$
\begin{aligned}
& \mathrm{E} \exp \left(-\alpha \bar{G}_{e_{q}}^{Z}+\mathrm{i} \beta \bar{Z}_{e_{q}}\right) \mathrm{E} \exp \left(-\alpha \underline{F}_{e_{q}}^{Z}+\mathrm{i} \beta \underline{Z}_{e_{q}}\right) \\
& \quad=\mathrm{E} \exp \left(-\alpha e_{\lambda+q}+\mathrm{i} \beta X_{e_{\lambda+q}}\right) \frac{1-\lambda /(\lambda+q)}{1-[\lambda /(\lambda+q+\alpha)] \mathrm{E} \exp \left(\mathrm{i} \beta X_{e_{\lambda+q+\alpha}}\right) \mathrm{Ee}^{\mathrm{i} \beta \xi}} .
\end{aligned}
$$


By conditioning on the value of $e_{\lambda+q}$ in the first factor, it is readily seen that this equals

$$
\begin{aligned}
\frac{q}{(\lambda+q+\alpha) / \mathrm{E} \exp \left(\mathrm{i} \beta X_{e_{\lambda+q+\alpha}}\right)-\lambda \mathrm{Ee}^{\mathrm{i} \beta \xi \xi}} & =\frac{q}{\lambda+q+\alpha+\Psi_{X}(\beta)-\lambda \mathrm{Ee}^{\mathrm{i} \beta \xi}} \\
& =\mathrm{E} \exp \left(-\alpha e_{q}+\mathrm{i} \beta Z_{e_{q}}\right),
\end{aligned}
$$

as desired.

Given Theorem 1, we can easily deduce the characteristics of the ladder height process of $Z$ in terms of those of $X$; as the notions are standard, we refer the reader to [7, p. 157] for definitions. The importance of this two-dimensional subordinator has recently been illustrated by Doney and Kyprianou [17].

The dual processes of $Z$ and $X$ are defined by $\hat{Z}=-Z$ and $\hat{X}=-X$, respectively.

Corollary 1. Under the assumptions of Theorem 1, we have, for $\alpha, \beta \geq 0$,

$$
\kappa_{Z}(\alpha, \beta)=\kappa_{X}(\lambda+\alpha, \beta)\left(1-\mathrm{Ee}^{-\beta H_{\mathrm{s}+}^{\alpha}}\left(\frac{\lambda}{\lambda+\alpha}\right)^{\tau_{\mathrm{s}+}^{\alpha}}\right)
$$

and

$$
\begin{aligned}
\hat{\kappa}_{Z}(\alpha, \beta) & =k \hat{\kappa}_{X}(\lambda+\alpha, \beta)\left(1-\mathrm{Ee}^{\beta H_{\mathrm{w}-}^{\alpha}}\left(\frac{\lambda}{\lambda+\alpha}\right)^{\tau_{\mathrm{w}-}^{\alpha}}\right) \\
& =k \frac{\alpha+\Psi_{Z}(-\mathrm{i} \beta)}{\kappa_{X}(\lambda+\alpha,-\beta)\left[1-\mathrm{Ee}^{\left.\beta H_{\mathrm{s}+}^{\alpha}(\lambda /(\lambda+\alpha))^{\tau_{\mathrm{s}+}^{\alpha}}\right]}\right.},
\end{aligned}
$$

where $k$ is some meaningless constant.

Proof. It suffices to note that $\kappa_{Z}(\alpha,-\mathrm{i} \beta) \hat{\kappa}_{Z}(\alpha, \mathrm{i} \beta)=k\left(\alpha+\Psi_{Z}(\beta)\right)$ by the Wiener-Hopf factorization for random walks, and to then analytically continue $\hat{\kappa}_{Z}$.

\section{Fluctuation theory with phase-type upward jumps}

In this section, we use the results of the previous section to study Lévy processes with so-called phase-type upward jumps and general downward jumps. According to the results of Section 2, $\left(\bar{G}_{e_{q}}^{Z}, \bar{Z}_{e_{q}}\right)$ can be written as the sum of $\left(\bar{G}_{e_{\lambda+q}}^{X}, \bar{X}_{e_{\lambda+q}}\right)$ and an (independent) random walk term. In this section, we choose $X$ and $Y$ in such a way that the transforms of both vectors can be computed explicitly. For this, we let $X$ be an arbitrary spectrally negative Lévy process and let $Y$ be a compound Poisson process (not necessarily a subordinator), independent of $X$, for which the upward jumps have a phase-type distribution. Recall that $Z=X+Y$.

Several applications motivate the investigation of fluctuations of Lévy processes with twosided jumps, e.g. with phase-type jumps in one direction. Focusing on their applications to options pricing, Asmussen et al. [5] and Mordecki [28] have recently studied these processes; see also [26]. The results are also relevant in the context of queues and risk processes; see [10] for a detailed discussion.

The exact form of the Lévy measure of $Y$, which facilitates the analysis, is specified in (2), below. Importantly, the upward jumps of $Y$ have a phase-type distribution. Recall that a phasetype distribution is the absorption time of a Markov process on a finite state space $E$. Its intensity matrix is determined by the $|E| \times|E|$ matrix $\boldsymbol{T}$, and its initial distribution is denoted by $\boldsymbol{\alpha}$. For more details on phase-type distributions, we refer the reader to [4, Section III.4]. We write $\boldsymbol{t}=-\boldsymbol{T} \mathbf{1}$, where $\mathbf{1}$ is the vector whose components all equal 1. Apart from their computational 
convenience, the most important property of phase-type distributions is that they are dense, in the sense of weak convergence, within the class of probability measures (although many phases may be needed to approximate a stable distribution, for instance).

As a consequence of this fact, an arbitrary Lévy process can be written as the limit of a sequence of Lévy processes with phase-type jumps (in the Skorokhod topology on $D\left(\mathbb{R}_{+}\right)$; see, e.g. [22, Chapter VI] for definitions). Both Asmussen et al. [5] and Mordecki [28] obtained expressions for the Laplace transform of $\bar{Z}_{e_{q}}$ if $Y$ is a compound Poisson process with only positive (phase-type) jumps.

While the class of processes that we analyze here is slightly more general, the main difference is that we calculate the Laplace transform of the joint distribution, $\left(\bar{G}_{e_{q}}^{Z}, \bar{Z}_{e_{q}}\right)$. In particular, if a Lévy process $Z$ has phase-type upward jumps, we can characterize the distribution of the epoch at which the supremum is attained; this is perhaps more surprising than the fact that we can find the distribution of $\bar{Z}_{e_{q}}$. This illustrates why Theorem 1 is interesting.

To the author's knowledge, the results of this section apply to any Lévy process for which this joint distribution is known. The only case for which results are available but not covered here is when $Z$ is a stable Lévy process, for which the Laplace transform of the (marginal) law of $\bar{Z}_{e_{q}}$ has been derived by C. C. Heyde, extending earlier work of D. A. Darling; see [15] for references and further details.

\subsection{The PRS factorization}

We begin with a detailed description of the process $Y$. Given a $K \in \mathbb{N}$, suppose that we have nonnegative random variables $\left\{A_{j}: j=1, \ldots, K\right\}$ and $\left\{B_{j}: j=1, \ldots, K\right\}$, where the distribution $\mathrm{P}_{B_{j}}$ of $B_{j}$ is phase type with representation $\left(E_{j}, \boldsymbol{\alpha}_{j}, \boldsymbol{T}_{j}\right)$. The distribution $\mathrm{P}_{-A_{j}}$ of $-A_{j}$ is general; the only restriction we impose is that $\mathrm{P}_{-A_{j}} * \mathrm{P}_{B_{j}}(\{0\})=0$ for all $j$, i.e. $A_{j}$ and $B_{j}$ do not both have an atom at 0 . Here ' $*$ ' denotes convolution. We assume that the process $Y$ is a compound Poisson process with Lévy measure given by

$$
\Pi_{Y}=\lambda \sum_{j=1}^{K} \pi_{j} \mathrm{P}_{B_{j}} * \mathrm{P}_{-A_{j}},
$$

where $\lambda \in(0, \infty)$ and $0 \leq \pi_{j} \leq 1$ with $\sum_{j} \pi_{j}=1$. In queueing theory (see, e.g. [4]), processes of this form arise naturally, and the $B_{j}$ can be interpreted as the service times and the $A_{j}$ as the interarrival times. Notice that $Y$ is a subordinator if and only if $\Pi_{Y}$ can be written in the form of (2) with $K=1$ and $A_{1} \equiv 0$.

Without loss of generality, we may assume that $E_{j}$ and $\boldsymbol{T}_{j}$ do not depend on $j$. Indeed, if $E_{j}$ has $m_{j}$ elements, we can construct an $E$ with $\sum_{j=1}^{K} m_{j}$ elements and $\boldsymbol{T}$ can then be chosen to be a block-diagonal matrix with the matrices $\boldsymbol{T}_{1}, \ldots, \boldsymbol{T}_{K}$ on its diagonal. The vectors $\boldsymbol{\alpha}_{j}$ are then filled out with 0 s, so that they consist of $K$ parts of lengths $m_{1}, \ldots, m_{K}$, with only the $j$ th part nonzero.

Fix some $q>0$; our first aim is to study the random walk $\left\{S_{n}^{q}\right\}$ introduced in Subsection 2.1, with generic step size distribution

$$
\mathrm{P}_{S_{1}^{q}}=\mathrm{P}_{\bar{X}_{e_{\lambda+q}}} * \mathrm{P}_{\underline{X}_{e_{\lambda+q}}} * \mathrm{P}_{\xi}
$$

(by PRS factorization), where $\mathrm{P}_{\xi}=\Pi_{Y} / \lambda$. We exclude the case where $Z$ is monotonic, so that $\mathrm{P}_{S_{1}^{q}}$ always assigns strictly positive probability to $\mathbb{R}_{+}$. In this case, there is no distinction between weak and strict ascending ladder heights, and we therefore write $\tau_{+}^{q}$ for $\tau_{\mathrm{w}+}^{q}=\tau_{\mathrm{s}+}^{q}$ throughout this section. 
Since $\bar{X}_{e_{\lambda+q}}$ is either degenerate or exponentially distributed, the law of $S_{1}^{q}$ can be written as

$$
\sum_{j} \pi_{j} \mathrm{P}_{B_{j}^{\prime}(q)} * \mathrm{P}_{A_{j}^{\prime}(q)},
$$

where $B_{j}^{\prime}(q)$ again has a phase-type distribution, say with representation $\left(E_{q}^{\prime}, \boldsymbol{\alpha}_{j}^{\prime}(q), \boldsymbol{T}_{q}^{\prime}\right)$. It is not hard to express this triple in terms of the original triple $\left(E, \boldsymbol{\alpha}_{j}, \boldsymbol{T}\right)$ : in fact $\left(E_{q}^{\prime}, \boldsymbol{\alpha}_{j}^{\prime}(q), \boldsymbol{T}_{q}^{\prime}\right)=$ $\left(E, \boldsymbol{\alpha}_{j}, \boldsymbol{T}\right)$ if $X$ is a negative subordinator; otherwise, $E_{q}^{\prime}$ can be chosen such that $\left|E_{q}^{\prime}\right|=|E|+1$ and the dynamics of the underlying Markov chain are unchanged, except that an additional state is visited before absorption. We set $\boldsymbol{t}_{q}^{\prime}=-\boldsymbol{T}_{q}^{\prime} \mathbf{1}$ and denote the identity matrix by $\boldsymbol{I}$.

Motivated by Theorem 1, the following lemma calculates the transform of the ladder variables $\left(H_{+}^{q}, \tau_{+}^{q}\right)$; recall that the random variables are only integrated over the subset $\left\{\tau_{+}^{q}<\infty\right\}$ of the probability space.

Lemma 1. Let $\rho \in(0,1)$ and $\beta \geq 0$. Then there exists some vector $\boldsymbol{\alpha}_{+}^{\rho, q}$ such that

$$
\mathrm{E} \rho^{\tau_{+}^{q}} \mathrm{e}^{-\beta H_{+}^{q}}=\boldsymbol{\alpha}_{+}^{\rho, q}\left(\beta \boldsymbol{I}-\boldsymbol{T}_{q}^{\prime}\right)^{-1} \boldsymbol{t}_{q}^{\prime} .
$$

Proof. The proof is similar to the proofs of Lemma VIII.5.1 and Proposition VIII.5.11 of [4]; the details are left to the reader.

The above lemma shows that it is of interest to be able to calculate $\boldsymbol{\alpha}_{+}^{\rho, q}$. Therefore, we generalize Theorem VIII.5.12 of [4] to the present setting. We omit a proof, as similar arguments apply here as do in [4]; the only differences are that we allow for $K>1$ and that the random walk can be killed at every step with probability $\rho$.

Proposition 2. The vector $\boldsymbol{\alpha}_{+}^{\rho, q}$ satisfies $\boldsymbol{\alpha}_{+}^{\rho, q}=\xi\left(\boldsymbol{\alpha}_{+}^{\rho, q}\right)$, where

$$
\xi\left(\boldsymbol{\alpha}_{+}^{\rho, q}\right)=\rho \sum_{j=1}^{K} \pi_{j} \boldsymbol{\alpha}_{j}^{\prime}(q) \int_{0}^{\infty} \mathrm{e}^{\left(\boldsymbol{T}_{q}^{\prime}+\boldsymbol{t}_{q}^{\prime} \boldsymbol{\alpha}_{+}^{\rho, q}\right) y} A_{j}^{\prime}(q)(\mathrm{d} y) .
$$

It can be computed as $\lim _{n \rightarrow \infty} \boldsymbol{\alpha}_{+}^{\rho, q}(n)$, where $\boldsymbol{\alpha}_{+}^{\rho, q}(0)=\mathbf{0}$ and $\boldsymbol{\alpha}_{+}^{\rho, q}(n)=\xi\left(\boldsymbol{\alpha}_{+}^{\rho, q}(n-1)\right)$ for $n \geq 1$.

The main result of this section follows by combining Theorem 1 with Lemma 1 and standard fluctuation identities; see, for instance, [7, Theorem VII.4]. If $\Phi_{X}(0)$ is the largest root of the equation $\psi_{X}(\beta)=0$, where $\psi_{X}(\beta)=-\Psi_{X}(-\mathrm{i} \beta)$, then we write $\Phi_{X}: \mathbb{R}_{+} \rightarrow\left[\Phi_{X}(0), \infty\right)$ for the inverse of the increasing function $\psi:\left[\Phi_{X}(0), \infty\right) \rightarrow \mathbb{R}_{+}$.

Since Theorem 1 applies directly if $X$ is monotonic, this scenario is excluded to focus on the most interesting case. For notational convenience, we write $\boldsymbol{\alpha}_{+}^{q}$ for $\boldsymbol{\alpha}_{+}^{q /(\lambda+q), q}$.

Theorem 2. Suppose that neither $X$ nor $Z$ is monotonic. Then, for $\alpha, \beta \geq 0$, we have

$$
\operatorname{Exp}\left(-\alpha \bar{G}_{e_{q}}^{Z}-\beta \bar{Z}_{e_{q}}\right)=\frac{\Phi_{X}(\lambda+q)\left[1-\boldsymbol{\alpha}_{+}^{q} \mathbf{1}\right]}{\left[\Phi_{X}(\lambda+q+\alpha)+\beta\right]\left[1-\boldsymbol{\alpha}_{+}^{q+\alpha}\left(\beta \boldsymbol{I}-\boldsymbol{T}_{q+\alpha}^{\prime}\right)^{-1} \boldsymbol{t}_{q+\alpha}^{\prime}\right]}
$$

and

$$
\mathrm{E} \exp \left(-\alpha \bar{F}_{e_{q}}^{Z}+\beta \underline{Z}_{e_{q}}\right)=\frac{q\left[\Phi_{X}(\lambda+q+\alpha)-\beta\right]\left[1+\boldsymbol{\alpha}_{+}^{q+\alpha}\left(\beta \boldsymbol{I}+\boldsymbol{T}_{q+\alpha}^{\prime}\right)^{-1} \boldsymbol{t}_{q+\alpha}^{\prime}\right]}{\left[q+\alpha+\Psi_{Z}(-\mathrm{i} \beta)\right] \Phi_{X}(\lambda+q)\left[1-\boldsymbol{\alpha}_{+}^{q} \mathbf{1}\right]} .
$$


Theorem 2 is an immediate consequence of Theorem 1 . We now formulate the corresponding analog of Corollary 1 . Note that the expression for $\kappa_{Z}(0, \beta)$ is already visible in the work of Mordecki [28]; here, we obtain a full description of $\kappa_{Z}$.

Corollary 2. Under the assumptions of Theorem 2, we have, for $\alpha, \beta \geq 0$,

$$
\kappa_{Z}(\alpha, \beta)=\left[\Phi_{X}(\lambda+\alpha)+\beta\right]\left[1-\boldsymbol{\alpha}_{+}^{\alpha}\left(\beta \boldsymbol{I}-\boldsymbol{T}_{\alpha}^{\prime}\right)^{-1} \boldsymbol{t}_{\alpha}^{\prime}\right]
$$

and

$$
\hat{\kappa}_{Z}(\alpha, \beta)=k \frac{\alpha+\Psi_{Z}(-\mathrm{i} \beta)}{\left[\Phi_{X}(\lambda+\alpha)-\beta\right]\left[1+\boldsymbol{\alpha}_{+}^{\alpha}\left(\beta \boldsymbol{I}+\boldsymbol{T}_{\alpha}^{\prime}\right)^{-1} \boldsymbol{t}_{\alpha}^{\prime}\right]},
$$

where $k$ is a meaningless constant.

\section{Perturbed risk models}

Let $X$ be an arbitrary Lévy process and $Y$ be a compound Poisson process with intensity $\lambda$ and generic (say integrable) positive jump $\xi$. In this section, we investigate the sum $Z=X+Y$, where the Lévy process $Z$ drifts to $-\infty$. Classical risk theory studies the supremum of $Z$ in the case that $X$ has negative drift, i.e. $X_{t}=-c t$ for some $c>\lambda \mathrm{E} \xi$. Its distribution is then given by the Pollaczek-Khinchin formula. In this analysis, a key role is played by ladder epochs and heights, i.e. quantities related to the event that $Z$ reaches a new record.

This section investigates the more general case where $X$ is an arbitrary Lévy process. In the literature, $Z$ is then known as a perturbed risk process; see [19], [20], [32], [33], and references therein. To analyze this model, the classical ladder epochs and heights are replaced by so-called modified ladder epochs and heights; these are related to the event that $Z$ reaches a new record as a result of a jump of $Y$.

More precisely, we define the modified ladder epoch $\chi$ as the first time a new supremum is reached by a jump of $Y$, i.e.

$$
\chi=\inf \left\{t>0: \Delta Y_{t}>\bar{Z}_{t-}-Z_{t-}\right\}
$$

For example, we have $\chi=T_{2}$ in Figure 1. As a convention, we define $\left(\bar{G}_{\chi-}^{Z}, \bar{Z}_{\chi_{-}}\right)$as $\left(\bar{G}_{\infty}^{Z}, \bar{Z}_{\infty}\right)$ on the event $\{\chi=\infty\}$.

The main difference between the present investigation of perturbed risk models and earlier work is that we are not only interested in Laplace transform for space-related variables such as $Z_{\chi}$, but also in the joint transform of time-space variables such as $\left(\chi, Z_{\chi}\right)$. This has not been studied before.

In Subsection 4.1, we derive some elementary time-space results for modified ladder variables, culminating in a Pollaczek-Khinchin formula for perturbed risk models. However, explicit formulae for the Laplace transforms cannot be derived. For this, we must impose the assumption that $Z$ is spectrally positive; this was also done in the above-cited articles on perturbed risk models. Subsection 4.2 investigates the spectrally positive case in detail. This results in a set of fluctuation identities that can be regarded as the perturbed risk analogs of the standard fluctuation identities (as given, for instance, by Bertoin [7, Theorem VII.4]). Interestingly, as a special case, we characterize the distribution of the modified ladder epoch $\chi$; this is one of the most natural quantities in the perturbed risk context, yet our results are new.

\subsection{Generalities}

In this subsection, we study the structure of a general perturbed risk model, i.e. we consider a general Lévy perturbation $X$. All results in this subsection are new in this general framework. 


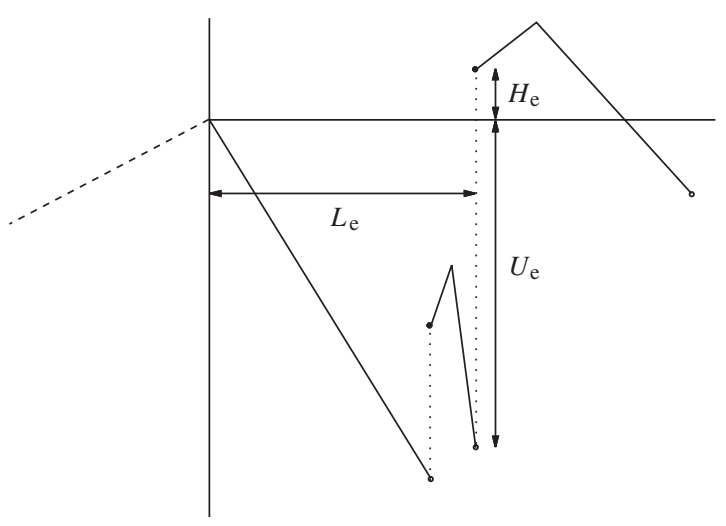

Figure 2: The excursion quantities in the proof of Proposition 3. The dashed line is the piece that is discarded under $\tilde{\mathrm{P}}$.

The following proposition is crucial for the analysis.

Proposition 3. Let $X$ be a general Lévy process (but not a subordinator) and let $Y$ be a compound Poisson subordinator. Then, for $Z=X+Y$,

1. $\left(\bar{G}_{\chi^{-}}^{Z}, \bar{Z}_{\chi^{-}}\right)$is independent of $\{\chi<\infty\}$;

2. $\left(\bar{G}_{\chi-}^{Z}, \bar{Z}_{\chi_{-}}\right)$is distributed as is $\left(\bar{G}_{\infty}^{Z}, \bar{Z}_{\infty}\right)$ given $\{\chi=\infty\}$; and

3. $\left(Z_{\chi}-\bar{Z}_{\chi_{-}}, \bar{Z}_{\chi_{-}-}-Z_{\chi_{-}}, \chi-\bar{G}_{\chi^{-}}^{Z}\right)$ is conditionally independent of $\left(\bar{G}_{\chi_{-}-}^{Z}, \bar{Z}_{\chi_{-}}\right)$given $\{\chi<\infty\}$.

Proof. We need some definitions related to the piecewise-linear (jump) process of Figure 1, in particular to its excursions. Let $\tilde{\mathrm{P}}$ denote the law of the piecewise-linear process that is constructed by discarding the first increasing piece (which may not be present if $X$ is a negative subordinator), and let $\tilde{\mathrm{E}}$ denote the corresponding expectation. Under $\tilde{\mathrm{P}}$, there are two possibilities for the process to cross the axis (strictly): the process either crosses it continuously or jumps over it. The first event is denoted by $\mathcal{X}$, as it is caused by fluctuations in $X$. We write $\mathcal{Y}$ for the second event. The probability of no crossing (i.e. no new record) is then given by $1-\tilde{\mathrm{P}}(\mathcal{X})-\tilde{\mathrm{P}}(\mathcal{Y})$. Moreover, by the strong Markov property, we have

$$
\mathrm{P}(\chi<\infty)=\frac{\tilde{\mathrm{P}}(y)}{1-\tilde{\mathrm{P}}(\chi)}
$$

On $\mathcal{y}$, we define the 'excursion lengths' $L_{\mathrm{e}}$, the 'excursion heights' $H_{\mathrm{e}}$, and the 'excursion undershoots' $U_{\mathrm{e}}$ as indicated in Figure 2 . We define $L_{\mathrm{e}}$ and $H_{\mathrm{e}}$ on $\mathcal{X}$ by considering the process formed by the maxima of the 'hats' as defined in Subsection 1.1. Let $H_{\mathrm{e}}$ be the first ascending ladder height of this process; note that this quantity is strictly positive (under $\tilde{\mathrm{P}}$ ) if $X$ is not a negative subordinator. We let $L_{\mathrm{e}}$ be the epoch at which the piecewise-linear process reaches $H_{\mathrm{e}}$. 
For $\alpha, \beta \geq 0$, by the strong Markov property,

$$
\begin{aligned}
\mathrm{E}\left[\exp \left(-\alpha \bar{G}_{\chi-}^{Z}-\beta \bar{Z}_{\chi-}\right) ; \chi<\infty\right] & \\
= & \mathrm{E} \exp \left(-\alpha \bar{G}_{e_{\lambda}}^{X}-\beta \bar{X}_{e_{\lambda}}\right) \tilde{\mathrm{P}}(\mathcal{y}) \\
& +\tilde{\mathrm{E}}\left[\mathrm{e}^{-\alpha L_{\mathrm{e}}-\beta H_{\mathrm{e}}} ; \chi\right] \mathrm{E}\left[\exp \left(-\alpha \bar{G}_{\chi-}^{Z}-\beta \bar{Z}_{\chi-}\right) ; \chi<\infty\right],
\end{aligned}
$$

from which we obtain

$$
\mathrm{E}\left[\exp \left(-\alpha \bar{G}_{\chi^{-}}^{Z}-\beta \bar{Z}_{\chi-}\right) ; \chi<\infty\right]=\mathrm{E} \exp \left(-\alpha \bar{G}_{e_{\lambda}}^{X}-\beta \bar{X}_{e_{\lambda}}\right) \frac{\tilde{\mathrm{P}}(\mathcal{y})}{1-\tilde{\mathrm{E}}\left[\mathrm{e}^{\left.-\alpha L_{\mathrm{e}}-\beta H_{\mathrm{e}} ; \mathcal{X}\right]}\right.} .
$$

Along the same lines, we can deduce that

$$
\mathrm{E}\left[\exp \left(-\alpha \bar{G}_{\infty}^{Z}-\beta \bar{Z}_{\infty}\right) ; \chi=\infty\right]=\mathrm{E} \exp \left(-\alpha \bar{G}_{e_{\lambda}}^{X}-\beta \bar{X}_{e_{\lambda}}\right) \frac{1-\tilde{\mathrm{P}}(\mathcal{X})-\tilde{\mathrm{P}}(\mathcal{Y})}{1-\tilde{\mathrm{E}}\left[\mathrm{e}^{-\alpha L_{\mathrm{e}}-\beta H_{\mathrm{e}}} ; \mathcal{X}\right]},
$$

meaning that

$$
\begin{aligned}
\frac{\mathrm{E}\left[\exp \left(-\alpha \bar{G}_{\chi-}^{Z}-\beta \bar{Z}_{\chi-}\right) ; \chi<\infty\right]}{\mathrm{P}(\chi<\infty)}= & \mathrm{E}\left[\exp \left(-\alpha \bar{G}_{\chi-}^{Z}-\beta \bar{Z}_{\chi-}\right) ; \chi<\infty\right] \\
& +\mathrm{E}\left[\exp \left(-\alpha \bar{G}_{\infty}^{Z}-\beta \bar{Z}_{\infty}\right) ; \chi=\infty\right]
\end{aligned}
$$

which is $\operatorname{Eexp}\left(-\alpha \bar{G}_{\chi^{-}}^{Z}-\beta \bar{Z}_{\chi_{-}}\right)$; this is the first claim. These calculations also show that

$$
\begin{aligned}
\frac{\mathrm{E}\left[\exp \left(-\alpha \bar{G}_{\infty}^{Z}-\beta \bar{Z}_{\infty}\right) ; \chi=\infty\right]}{\mathrm{P}(\chi=\infty)}= & \mathrm{E}\left[\exp \left(-\alpha \bar{G}_{\chi-}^{Z}-\beta \bar{Z}_{\chi-}\right) ; \chi<\infty\right] \\
& +\mathrm{E}\left[\exp \left(-\alpha \bar{G}_{\infty}^{Z}-\beta \bar{Z}_{\infty}\right) ; \chi=\infty\right],
\end{aligned}
$$

which is the second claim.

For the third claim, a variant of this argument can be used to see that, for $\alpha, \beta, \gamma, \delta, \varepsilon \geq 0$,

$$
\begin{aligned}
& \mathrm{E}\left[\exp \left(-\alpha \bar{G}_{\chi-}^{Z}-\beta \bar{Z}_{\chi-}\right) \exp \left(-\gamma\left[Z_{\chi}-\bar{Z}_{\chi-}\right]\right)\right. \\
& \left.\quad \times \exp \left(-\delta\left[\bar{Z}_{\chi-}-Z_{\chi-}\right]\right) \exp \left(-\varepsilon\left[\chi-\bar{G}_{\chi-}^{Z}\right]\right) \mid \chi<\infty\right] \\
& \quad=\operatorname{E} \exp \left(-\alpha \bar{G}_{e_{\lambda}}^{X}-\beta \bar{X}_{e_{\lambda}}\right) \frac{1-\tilde{\mathrm{P}}(\mathcal{X})}{1-\tilde{\mathrm{E}}\left[\mathrm{e}^{-\alpha L_{\mathrm{e}}-\beta H_{\mathrm{e}}} ; \mathcal{X}\right]} \frac{\tilde{\mathrm{E}}\left[\mathrm{e}^{-\gamma H_{\mathrm{e}}} \mathrm{e}^{-\delta U_{\mathrm{e}}} \mathrm{e}^{-\varepsilon L_{\mathrm{e}}} ; y\right]}{\tilde{\mathrm{P}}(\mathcal{y})} .
\end{aligned}
$$

This completes the proof of the proposition.

The formula in the following theorem can be viewed as a generalized Pollaczek-Khinchin formula for perturbed risk models. It is a consequence of the preceding proposition and the observation that, by the strong Markov property,

$\operatorname{Eexp}\left(-\alpha \bar{G}_{\infty}^{Z}-\beta \bar{Z}_{\infty}\right)$

$$
=\frac{\mathrm{E}\left[\exp \left(-\alpha \bar{G}_{\infty}^{Z}-\beta \bar{Z}_{\infty}\right) ; \chi=\infty\right]}{1-\mathrm{E}\left[\exp \left(-\alpha \bar{G}_{\chi^{-}}^{Z}-\beta \bar{Z}_{\chi^{-}}\right) ; \chi<\infty\right] \mathrm{E}\left[\exp \left(-\alpha\left[\chi-\bar{G}_{\chi^{-}}^{Z}\right]-\beta\left[Z_{\chi}-\bar{Z}_{\chi-}\right]\right) \mid \chi<\infty\right]} .
$$


Theorem 3. Under the assumptions of Proposition 3, we have, for $\alpha, \beta \geq 0$, provided that $Z$ drifts to $-\infty$,

$$
\begin{aligned}
& \mathrm{E} \exp \left(-\alpha \bar{G}_{\infty}^{Z}-\beta \bar{Z}_{\infty}\right) \\
& =\frac{\mathrm{P}(\chi=\infty) \mathrm{E} \exp \left(-\alpha \bar{G}_{\chi-}^{Z}-\beta \bar{Z}_{\chi-}\right)}{1-\mathrm{P}(\chi<\infty) \mathrm{E} \exp \left(-\alpha \bar{G}_{\chi^{-}}^{Z}-\beta \bar{Z}_{\chi-}\right) \mathrm{E}\left[\exp \left(-\alpha\left[\chi-\bar{G}_{\chi^{-}}^{Z}\right]-\beta\left[Z_{\chi}-\bar{Z}_{\chi-}\right]\right) \mid \chi<\infty\right]}
\end{aligned}
$$

\subsection{Spectrally positive $Z$}

In this subsection, we analyze the case where $Z$ has only positive jumps. We work under the assumptions of Proposition 3, but additionally assume that $\mathrm{E} Z_{1}<0$ (in particular, $Z$ is integrable) and that the Lévy measure of $Z$ vanishes on the negative half-line. It turns out that the transforms of the previous section can then be computed. As an aside, we remark that perturbed risk models with positive jumps are related to M/G/1 queueing systems with a second service; see [13].

Throughout, we exclude the case where $X$ is a negative subordinator, i.e. where $X$ has negative drift; the analysis is then classical. By doing so, the standard fluctuation identities (Theorem VII.4 of [7]) apply to both $X$ and $Z$. We use these identities without further reference.

Perturbed risk models under spectral positivity have been recently examined by Huzak et al. [20]. The main difference between this subsection and [20] is the fact that we are interested in examining the time-space domain (e.g. the transform of $\left(\chi, Z_{\chi}\right)$ ), as opposed to only the space domain. Consequently, our results generalize those of Huzak et al. [20], with the following caveat. Huzak et al. allowed $Y$ to be a general subordinator, not necessarily of the compound Poisson type. Therefore, the perturbed risk models studied here are slightly less general. However, since any subordinator can be approximated by compound Poisson subordinators and the modified ladder epochs form a discrete set (see [20]), our results must also hold in the general case. Since the approximation argument required for a rigorous proof is not in the spirit of this paper, we do not address this issue here. Instead, we shall content ourselves with writing the main result (Theorem 4) in a form that does not rely on $Y$ being compound Poisson, although this assumption is essential for the proof.

Our analysis is based on the Wiener-Hopf theory for Markov additive processes. Indeed, the piecewise-linear embedded process is closely related to a discrete-time Markov additive process (see, e.g. [4, Chapter XI] for the definition) with defective step size distributions. To see this, note that each 'hat' (see Subsection 1.1) contains three important points: its starting point (labeled '1'), its maximum (labeled '2'), and (immediately before) its endpoint (labeled ' 3 '). Let us now fix some $\alpha \geq 0$. Equation (VI.1) of [7] implies that, for $q>0$ and $\beta \in \mathbb{R}$,

$$
\mathrm{E} \exp \left(-\alpha \bar{G}_{e_{q}}^{X}+\mathrm{i} \beta \bar{X}_{e_{q}}\right)=\mathrm{E} \exp \left(-\alpha \bar{G}_{e_{q}}^{X}\right) \mathrm{E} \exp \left(\mathrm{i} \beta \bar{X}_{e_{q+\alpha}}\right)
$$

and similarly for the joint distribution of $\left(\underline{F}_{e_{q}}^{X}, \underline{X}_{e_{q}}\right)$. In other words, since $\alpha$ is fixed, the joint distribution (in time and space) of the increment from point 1 to point 2 , as well as from point 2 to point 3 , can be interpreted as a defective marginal distribution in space. Hence, a 'killing mechanism' has been introduced, which allows us to study joint distributions in time and space by applying (known) results on processes with given marginal (space) distributions. 
More precisely, we define a Markov additive process in discrete time, $\left\{\left(J_{n}, S_{n}\right)\right\}$, as the Markov process with state space $\{1,2,3\} \times \mathbb{R}$, characterized by the transform matrix

$$
\boldsymbol{F}(\alpha, \beta)=\left(\begin{array}{ccc}
0 & \mathrm{E} \exp \left(-\alpha \bar{G}_{e_{\lambda}}^{X}\right) \operatorname{Eexp}\left(\mathrm{i} \beta \bar{X}_{e_{\lambda+\alpha}}\right) & 0 \\
0 & 0 & \mathrm{E} \exp \left(-\alpha \underline{F}_{e_{\lambda}}^{X}\right) \operatorname{Eexp}\left(\mathrm{i} \beta \underline{X}_{e_{\lambda+\alpha}}\right) \\
\mathrm{Ee}^{\mathrm{i} \beta \xi} & 0 & 0
\end{array}\right)
$$

That is, $S_{0}=0$ and $J_{n}$ is deterministic given $J_{0}$ : in every time slot, it jumps from $i$ to $i+1$ unless $i=3$; then it jumps back to 1 . If $J_{n-1}=1$ (i.e. point $n-1$ corresponds to the beginning of a 'hat'), then the process is killed with probability $1-\operatorname{Eexp}\left(-\alpha \bar{G}_{e_{\lambda}}^{X}\right)$; otherwise, we set $S_{n}=S_{n-1}+\eta_{n-1}$, where $\eta_{n-1}$ is independent of $S_{n-1}$ and distributed as is $\bar{X}_{e_{\lambda+\alpha}}$. The cases $J_{n-1}=2$ and $J_{n-1}=3$ are similar, except for the absence of killing in the latter case. We also write

$$
\tau_{+}=\inf \left\{n>0: S_{n}>0\right\}, \quad \tau_{-}=\inf \left\{n>0: S_{n} \leq 0\right\} .
$$

Expressions such as $\mathrm{P}_{2}\left(J_{\tau_{+}}=2\right)$ should be understood as $\mathrm{P}\left(J_{\tau_{+}}=2, \tau_{+}<\infty \mid J_{0}=2\right)$, and similarly for $\mathrm{E}_{2}$.

In the Wiener-Hopf theory for Markov additive processes, an important role is played by the time-reversed process. To define this process, we introduce the Markov chain $\hat{J}$, for which the transitions are deterministic: it jumps from 3 to 2, from 2 to 1 , and from 1 to 3 . Hence, it jumps in the opposite direction to $J$. We set $\hat{S}_{0}=0$ and define the transition structure of the time-reversed Markov additive process $(\hat{J}, \hat{S})$ as follows. If $\hat{J}_{n-1}=2$ then the process is killed with probability $1-\operatorname{E} \exp \left(-\alpha \bar{G}_{e_{2}}^{X}\right)$; otherwise, we set $\hat{S}_{n}=\hat{S}_{n-1}+\hat{\eta}_{n-1}$, where $\hat{\eta}_{n-1}$ is independent of $\hat{S}_{n-1}$ and distributed as is $\bar{X}_{e_{\lambda+\alpha}}$. Similarly, if $\hat{J}_{n-1}=3$ then the process is killed with probability $1-\mathrm{E} \exp \left(-\alpha \underline{F}_{e_{\lambda}}^{X}\right)$; otherwise, the increment is distributed as is $\underline{X}_{e_{\lambda+\alpha}}$. If $\hat{J}_{n-1}=1$ then the increment is distributed as is $\xi>0$. The quantities $\hat{\tau}_{+}$and $\hat{\tau}_{-}$are defined as the ladder epochs for $\hat{S}$. We write $\hat{\mathrm{P}}_{2}$ for the conditional distribution given that $\hat{J}_{0}=2$.

Recalling that the dependence on $\alpha$ is 'absorbed' in the killing mechanism, we define

$$
G_{+}^{(k, \ell)}(\alpha, \beta)=\mathrm{E}_{k}\left[\exp \left(\mathrm{i} \beta S_{\tau_{+}}\right) ; J_{\tau_{+}}=\ell\right]
$$

and

$$
\hat{G}_{-}^{(k, \ell)}(\alpha, \beta)=\hat{\mathrm{E}}_{k}\left[\exp \left(\mathrm{i} \beta \hat{S}_{\hat{\tau}_{-}}\right) ; \hat{J}_{\hat{\tau}_{-}}=j\right] .
$$

Note that $G_{+}^{(2,2)}=\tilde{\mathrm{E}}\left[\mathrm{e}^{-\alpha L_{\mathrm{e}}+\mathrm{i} \beta H_{\mathrm{e}}} ; \mathcal{X}\right]$ in the notation of the proof of Proposition 3, and similarly $G_{+}^{(2,1)}=\tilde{\mathrm{E}}\left[\mathrm{e}^{-\alpha L_{\mathrm{e}}+\mathrm{i} \beta H_{\mathrm{e}}} ; y\right]$.

The Wiener-Hopf factorization for Markov additive processes (see, e.g. [4, Theorem XI.2.12] or [31, Theorem 5.2]) states that $\boldsymbol{I}-\boldsymbol{F}(\alpha, \beta)$ equals

$$
\left(\begin{array}{ccc}
1 & 0 & 0 \\
-\hat{G}_{-}^{(1,2)} & 1-\hat{G}_{-}^{(2,2)} & -\hat{G}_{-}^{(3,2)} \\
0 & 0 & 1
\end{array}\right)\left(\begin{array}{ccc}
1 & -\mathrm{E} \exp \left(-\alpha \bar{G}_{e_{\lambda}}^{X}+\mathrm{i} \beta \bar{X}_{e_{\lambda}}\right) & 0 \\
-G_{+}^{(2,1)} & 1-G_{+}^{(2,2)} & 0 \\
-\mathrm{Ee}^{\mathrm{i} \beta \xi} & 0 & 1
\end{array}\right),
$$

where the arguments, $\alpha$ and $\beta$, of $G_{+}$and $\hat{G}_{-}$are suppressed for notational convenience.

We start by computing the first matrix. Note that $\hat{G}_{-}^{(3,2)}(\alpha, \beta)=\operatorname{E} \exp \left(-\alpha \underline{F}_{e_{\lambda}}^{X}+\mathrm{i} \beta \underline{X}_{e_{\lambda}}\right)$, so two terms remain. Recall that $\Phi_{-X}$ is the inverse of the function $\beta \mapsto \psi_{-X}(\beta)=-\Psi_{-X}(-\mathrm{i} \beta)$, and similarly for $\Phi_{-Z}$. 
Proposition 4. For $\beta \in \mathbb{R}$, we have

$$
\hat{G}_{-}^{(1,2)}(\alpha, \beta)=\mathrm{Ee}^{-\Phi_{-Z}(\alpha) \xi} \frac{\Phi_{-X}(\lambda)}{\Phi_{-X}(\lambda+\alpha)+\mathrm{i} \beta}
$$

and

$$
\hat{G}_{-}^{(2,2)}(\alpha, \beta)=\frac{\Phi_{-X}(\lambda+\alpha)-\Phi_{-Z}(\alpha)}{\Phi_{-X}(\lambda+\alpha)+\mathrm{i} \beta} .
$$

Proof. We start with $\hat{G}_{-}^{(2,2)}$. By 'gluing together' the transitions $2 \rightarrow 1$ and $1 \rightarrow 3$, we see that the killing probability of going from 2 to itself now equals $\lambda /(\lambda+\alpha)$, and the distribution of a jump from 2 to itself can be written as $\xi+\bar{X}_{e_{\lambda+\alpha}}-e_{\Phi_{-X}(\lambda+\alpha)}$, where all three components are independent. Therefore, by standard results on random walks (see, e.g. Lemma I.4 of [31]), we have

$$
\hat{G}_{-}^{(2,2)}(\alpha, \beta)=\hat{\mathrm{E}}_{2}\left(\frac{\lambda}{\lambda+\alpha}\right)^{\hat{\tau}_{-}} \mathrm{e}^{\mathrm{i} \beta \hat{S}_{\hat{\tau}_{-}}}=\frac{\Phi_{-X}(\lambda+\alpha)}{\Phi_{-X}(\lambda+\alpha)+\mathrm{i} \beta} \hat{\mathrm{E}}_{2}\left(\frac{\lambda}{\lambda+\alpha}\right)^{\hat{\tau}_{-}},
$$

and it remains to calculate the mean, which we write as $\eta_{\alpha}$. To do so, we repeat the argument that led to Theorem 1, but now for the minimum and in terms of $\eta_{\alpha}$. We see that $\mathrm{E} \mathrm{e}^{\mathrm{i} \beta \underline{Z}_{e_{\alpha}}}$ equals

$$
\begin{aligned}
\frac{\Phi_{-Z}(\alpha)}{\Phi_{-Z}(\alpha)+\mathrm{i} \beta} & =\frac{\Phi_{-X}(\lambda+\alpha)}{\Phi_{-X}(\lambda+\alpha)+\mathrm{i} \beta} \frac{1-\eta_{\alpha}}{1-\eta_{\alpha} \Phi_{-X}(\lambda+\alpha) /\left(\Phi_{-X}(\lambda+\alpha)+\mathrm{i} \beta\right)} \\
& =\frac{\left(1-\eta_{\alpha}\right) \Phi_{-X}(\lambda+\alpha)}{\left(1-\eta_{\alpha}\right) \Phi_{-X}(\lambda+\alpha)+\mathrm{i} \beta},
\end{aligned}
$$

implying that $1-\eta_{\alpha}=\Phi_{-Z}(\alpha) / \Phi_{-X}(\lambda+\alpha)$.

Now we study $\hat{G}_{-}^{(1,2)}$. A descending ladder epoch occurs either at the first time that $\hat{J}$ visits 2 , or during subsequent visits. The contribution to $\hat{G}_{-}^{(1,2)}$ of the first term is

$$
\begin{aligned}
\hat{\mathrm{E}}_{1}\left[\mathrm{e}^{\mathrm{i} \beta \hat{S}_{2}} ; \hat{S}_{2}<0\right] & =\mathrm{E} \exp \left(-\alpha \underline{F}_{e_{\lambda}}^{X}\right) \mathrm{E}\left[\exp \left(\mathrm{i} \beta\left(\xi+\underline{X}_{e_{\lambda+\alpha}}\right)\right) ; \xi+\underline{X}_{e_{\lambda+\alpha}}<0\right] \\
& =\int_{0}^{\infty} \Phi_{-X}(\lambda) \mathrm{e}^{-\left(\Phi_{-X}(\lambda+\alpha)+\mathrm{i} \beta\right) t} \mathrm{E}\left[\mathrm{e}^{\mathrm{i} \beta \xi} ; \xi<t\right] \mathrm{d} t \\
& =\mathrm{Ee}^{\mathrm{i} \beta \xi} \int_{0}^{\xi} \Phi_{-X}(\lambda) \mathrm{e}^{-\left(\Phi_{-X}(\lambda+\alpha)+\mathrm{i} \beta\right) t} \mathrm{~d} t \\
& =\frac{\Phi_{-X}(\lambda)}{\Phi_{-X}(\lambda+\alpha)+\mathrm{i} \beta} \mathrm{Ee}^{-\Phi_{-X}(\lambda+\alpha) \xi}
\end{aligned}
$$

To compute the contribution to $\hat{G}_{-}^{(1,2)}$ of paths for which $\hat{S}_{2}$ is positive, we apply results of Arjas and Speed [1] on random walks with a random initial point. We also use their notation.

Using the previously computed expression for $\hat{G}_{-}^{(2,2)}$ (again, the transform depends on $\alpha$ through the killing mechanism), we define

$$
\begin{aligned}
\bar{w}_{z-}(\beta) & =\frac{1}{1-\hat{G}_{-}^{(2,2)}(\alpha, \beta)}=\frac{1}{1-\left(\Phi_{-X}(\lambda+\alpha)-\Phi_{-Z}(\alpha)\right) /\left(\Phi_{-X}(\lambda+\alpha)+\mathrm{i} \beta\right)} \\
& =1+\frac{\Phi_{-X}(\lambda+\alpha)-\Phi_{-Z}(\alpha)}{\Phi_{-Z}(\alpha)+\mathrm{i} \beta} .
\end{aligned}
$$


As in [1], define the projection operator $\mathcal{P}$ acting on a Fourier transform $f(\beta)=\int_{\mathbb{R}} \mathrm{e}^{\mathrm{i} \beta x} F(\mathrm{~d} x)$ as $\mathcal{P} f(\beta)=\int_{(-\infty, 0]} \mathrm{e}^{\mathrm{i} \beta x} F(\mathrm{~d} x)$. Theorem 1(b) of [1] shows that the second contribution to $\hat{G}_{-}^{(1,2)}$ equals

$$
\frac{1}{\bar{w}_{z-}(\beta)} \mathcal{P}\left[\mathrm{E}^{-\alpha \underline{F}^{X}\left(e_{\lambda}\right)} \mathrm{E}\left[\mathrm{e}^{\mathrm{i} \beta(\xi+\underline{X}(\lambda+\alpha))} ; \xi+\underline{X}(\lambda+\alpha)>0\right] \bar{w}_{z-}(\beta)\right] .
$$

Similar reasoning as above shows that

$$
\begin{aligned}
\hat{\mathrm{E}}_{1}\left[\mathrm{e}^{\mathrm{i} \beta \hat{S}_{2}} ; \hat{S}_{2}>0\right] & =\mathrm{E} \exp \left(-\alpha \underline{F}_{e_{\lambda}}^{X}\right) \mathrm{E}\left[\exp \left(\mathrm{i} \beta\left(\xi+\underline{X}_{e_{\lambda+\alpha}}\right)\right) ; \xi+\underline{X}_{e_{\lambda+\alpha}}>0\right] \\
& =\Phi_{-X}(\lambda) \int_{0}^{\infty} \mathrm{e}^{-\left(\Phi_{-X}(\lambda+\alpha)+\mathrm{i} \beta\right) t} \mathrm{E}\left[\mathrm{e}^{\mathrm{i} \beta \xi} ; \xi>t\right] \mathrm{d} t \\
& =\Phi_{-X}(\lambda) \frac{\mathrm{E} \mathrm{e}^{\mathrm{i} \beta \xi}-\mathrm{Ee}^{-\Phi_{-X}(\lambda+\alpha) \xi}}{\Phi_{-X}(\lambda+\alpha)+\mathrm{i} \beta} .
\end{aligned}
$$

As this is the transform of a positive random variable, the first observation in the proof of Corollary 1 of [1] shows that

$$
\begin{aligned}
\mathcal{P}\left[\frac{\Phi_{-X}(\lambda+\alpha)-\Phi_{-Z}(\alpha)}{\Phi_{-Z}(\alpha)+\mathrm{i} \beta} \hat{\mathrm{E}}_{1}\left[\mathrm{e}^{\mathrm{i} \beta \hat{S}_{2}} ; \hat{S}_{2}>0\right]\right] \\
\quad=\frac{\Phi_{-X}(\lambda+\alpha)-\Phi_{-Z}(\alpha)}{\Phi_{-Z}(\alpha)+\mathrm{i} \beta} \hat{\mathrm{E}}_{1}\left[\mathrm{e}^{-\Phi_{-Z}(\alpha) \hat{S}_{2}} ; \hat{S}_{2}>0\right] .
\end{aligned}
$$

Therefore, (4) equals

$$
\begin{gathered}
\Phi_{-X}(\lambda) \frac{\mathrm{Ee}^{-\Phi_{-Z}(\alpha) \xi}-\mathrm{Ee}^{-\Phi_{-X}(\lambda+\alpha) \xi}}{\Phi_{-X}(\lambda+\alpha)-\Phi_{-Z}(\alpha)} \frac{\left(\Phi_{-X}(\lambda+\alpha)-\Phi_{-Z}(\alpha)\right) /\left(\Phi_{-Z}(\alpha)+\mathrm{i} \beta\right)}{1+\left(\Phi_{-X}(\lambda+\alpha)-\Phi_{-Z}(\alpha)\right) /\left(\Phi_{-Z}(\alpha)+\mathrm{i} \beta\right)} \\
=\frac{\Phi_{-X}(\lambda)}{\Phi_{-X}(\lambda+\alpha)+\mathrm{i} \beta}\left[\mathrm{Ee}^{-\Phi_{-Z}(\alpha) \xi}-\mathrm{Ee}^{-\Phi_{-X}(\lambda+\alpha) \xi}\right] .
\end{gathered}
$$

Summing the two contributions completes the proof of the claim and, hence, that of the proposition.

With the preceding proposition at our disposal, the Wiener-Hopf factorization yields

$$
G_{+}^{(2,1)}(\alpha, \beta)=\Phi_{-X}(\lambda) \frac{\mathrm{Ee}^{\mathrm{i} \beta \xi}-\mathrm{Ee}^{-\Phi_{-Z}(\alpha) \xi}}{\Phi_{-Z}(\alpha)+\mathrm{i} \beta}
$$

and

$$
1-G_{+}^{(2,2)}(\alpha, \beta)=\frac{\Phi_{-X}(\lambda+\alpha)+\mathrm{i} \beta-\Phi_{-X}(\lambda) \mathrm{Ee}^{-\Phi_{Z}(\alpha) \xi} \mathrm{E} \exp \left(-\alpha \bar{G}_{e_{\lambda}}^{X}+\mathrm{i} \beta \bar{X}_{e_{\lambda}}\right)}{\Phi_{-Z}(\alpha)+\mathrm{i} \beta},
$$

where $\operatorname{E} \exp \left(-\alpha \bar{G}_{e_{\lambda}}^{X}+\mathrm{i} \beta \bar{X}_{e_{\lambda}}\right)$ is explicitly known in terms of $\Phi_{-X}$. From these expressions, by choosing $\alpha=\beta=0$ we obtain $\tilde{\mathrm{P}}(\mathcal{X})=1+\left(\Phi_{-X}(\lambda) / \lambda\right) \mathrm{E} X_{1}$ and $\tilde{\mathrm{P}}(\mathcal{Y})=\Phi_{-X}(\lambda) \mathrm{E} \xi$. In particular, $1-\tilde{\mathrm{P}}(\mathcal{X})-\tilde{\mathrm{P}}(\mathcal{Y})=-\left(\Phi_{-X}(\lambda) / \lambda\right) \mathrm{E} Z_{1}$.

Our next goal is to characterize distributions related to modified ladder epochs and heights, which is the main result of this subsection.

Theorem 4. Let $X$ be a general spectrally positive Lévy process (which is not monotonic), and let $Y$ be a compound Poisson subordinator. Suppose that both $X$ and $Y$ are integrable, and that $Z=X+Y$ satisfies $\mathrm{E} Z_{1}<0$. 
1. For $\alpha, \beta \geq 0$, we have

$$
\begin{aligned}
\mathrm{E} \exp \left(-\alpha \bar{G}_{\chi-}^{Z}-\beta \bar{Z}_{\chi-}\right) & =\mathrm{E}\left[\exp \left(-\alpha \bar{G}_{\chi-}^{Z}-\beta \bar{Z}_{\chi-}\right) \mid \chi<\infty\right] \\
& =\mathrm{E}\left[\exp \left(-\alpha \bar{G}_{\infty}^{Z}-\beta \bar{Z}_{\infty}\right) \mid \chi=\infty\right] \\
& =-\mathrm{E} X_{1} \frac{\Phi_{-Z}(\alpha)-\beta}{\alpha-\psi_{-X}(\beta)-\psi_{-Y}\left(\Phi_{-Z}(\alpha)\right)},
\end{aligned}
$$

which should be interpreted as $-\mathrm{E} X_{1} / \psi_{-Z}^{\prime}(\beta)$ for $\beta=\Phi_{-Z}(\alpha)$. In particular, $\bar{Z}_{\chi-}$ has the same distribution as $\bar{X}_{\infty}$.

2. For $\alpha, \beta \geq 0$, we have

$$
\mathrm{E}\left[\exp \left(-\alpha\left[\chi-\bar{G}_{\chi-}^{Z}\right]-\beta\left[Z_{\chi}-\bar{Z}_{\chi-}\right]\right) \mid \chi<\infty\right]=\frac{1}{\operatorname{E} Y_{1}} \frac{\psi_{-Y}(\beta)-\psi_{-Y}\left(\Phi_{-Z}(\alpha)\right)}{\Phi_{-Z}(\alpha)-\beta},
$$

which should be interpreted as $-\psi_{-Y}^{\prime}(\beta) / \mathrm{E} Y_{1}$ for $\beta=\Phi_{-Z}(\alpha)$. In particular, for $y, z>0$,

$$
\mathrm{P}\left(Z_{\chi}-\bar{Z}_{\chi-}>x, \bar{Z}_{\chi-}-Z_{\chi-}>y \mid \chi<\infty\right)=\frac{1}{\mathrm{E} \xi} \int_{x+y}^{\infty} \mathrm{P}(\xi>u) \mathrm{d} u .
$$

3. For $\alpha, \beta \geq 0$, we have

$$
\mathrm{E}\left[\mathrm{e}^{-\alpha \chi-\beta Z_{\chi}} ; \chi<\infty\right]=\frac{\psi_{-Y}(\beta)-\psi_{-Y}\left(\Phi_{-Z}(\alpha)\right)}{\alpha-\psi_{-X}(\beta)-\psi_{-Y}\left(\Phi_{-Z}(\alpha)\right)},
$$

which should be interpreted as $-\psi_{-Y}^{\prime}(\beta) / \psi_{-Z}^{\prime}(\beta)$ for $\beta=\Phi_{-Z}(\alpha)$. In particular,

$$
\mathrm{P}(\chi<\infty)=1-\mathrm{E} Z_{1} / \mathrm{E} X_{1} .
$$

Proof. To compute the transform of the joint distribution of $\left(\bar{G}_{\chi_{-}}^{Z}, \bar{Z}_{\chi_{-}-}\right)$, we use elements of the proof of Proposition 3: we have, in particular,

$$
\begin{aligned}
& \mathrm{E} \exp \left(-\alpha \bar{G}_{\chi-}^{Z}-\beta \bar{Z}_{\chi-}\right) \\
& =\mathrm{E} \exp \left(-\alpha \bar{G}_{e_{\lambda}}^{X}-\beta \bar{X}_{e_{\lambda}}\right) \frac{1-\tilde{\mathrm{P}}(\mathcal{X})}{1-G_{+}^{(2,2)}(\alpha, \mathrm{i} \beta)} \\
& =-\mathrm{E} X_{1} \frac{\left(\Phi_{-X}(\lambda) / \lambda\right)\left[\Phi_{-Z}(\alpha)-\beta\right]}{\left(\Phi_{-X}(\lambda+\alpha)-\beta\right) / \mathrm{E} \exp \left(-\alpha \bar{G}_{e_{\lambda}}^{X}-\beta \bar{X}_{e_{\lambda}}\right)-\Phi_{-X}(\lambda) \mathrm{Ee}^{-\Phi_{-Z}(\alpha) \xi}},
\end{aligned}
$$

from which the first claim follows.

The second claim is a consequence of the fact that the transform equals $G_{+}^{(2,1)}(\alpha, \beta) / \tilde{\mathrm{P}}(\mathcal{y})$. The second statement follows by choosing $\alpha=0$ and noting that

$$
\mathrm{P}\left(\bar{Z}_{\chi_{-}}-Z_{\chi-}>x \mid Z_{\chi}-\bar{Z}_{\chi-}=y, \chi<\infty\right)=\mathrm{P}(\xi>x+y \mid \xi>y) .
$$

The third claim is obtained from the identity

$$
\begin{aligned}
\mathrm{E}\left[\mathrm{e}^{-\alpha \chi-\beta Z_{\chi}} \mid \chi<\infty\right]= & \mathrm{E}\left[\exp \left(-\alpha \bar{G}_{\chi-}^{Z}-\beta \bar{Z}_{\chi-}\right) \mid \chi<\infty\right] \\
& \times \mathrm{E}\left[\exp \left(-\alpha\left[\chi-\bar{G}_{\chi^{-}}^{Z}\right]-\beta\left[Z_{\chi}-\bar{Z}_{\chi-}\right]\right) \mid \chi<\infty\right]
\end{aligned}
$$

and (3). This completes the proof. 
Let us now calculate the transform of $\left(G_{\infty}^{Z}, \bar{Z}_{\infty}\right)$ using Theorem 3: for $\alpha, \beta \geq 0$, we have

$$
\begin{aligned}
& \mathrm{E} \exp \left(-\alpha \bar{G}_{\infty}^{Z}-\beta \bar{Z}_{\infty}\right) \\
& \quad=-\mathrm{E} Z_{1} \frac{\Phi_{-Z}(\alpha)-\beta}{\alpha-\psi_{-X}(\beta)-\psi_{-Y}\left(\Phi_{-Z}(\alpha)\right)}\left(1-\frac{\psi_{-Y}(\beta)-\psi_{-Y}\left(\Phi_{-Z}(\alpha)\right)}{\alpha-\psi_{-X}(\beta)-\psi_{-Y}\left(\Phi_{-Z}(\alpha)\right)}\right)^{-1} \\
& \quad=\frac{-\mathrm{E} Z_{1}\left[\Phi_{-Z}(\alpha)-\beta\right]}{\alpha-\psi_{-X}(\beta)-\psi_{-Y}(\beta)},
\end{aligned}
$$

in accordance with the standard fluctuation identity.

Note that Theorem 4.7 of [20] is recovered upon combining the particular-case results of this theorem with Proposition 3, at least if $Y$ is compound Poisson. There is also another way to see that $\mathrm{P}\left(\bar{Z}_{\chi-} \leq x \mid \chi<\infty\right)=\mathrm{P}\left(\bar{X}_{\infty} \leq x\right)$. Indeed, one can 'cut away' certain pieces of the path of $Z$; Schmidli [33] made this precise by time reversal of $Z$. However, his argument cannot be used to find the distribution of $\bar{G}_{\chi^{-}}^{Z}$.

We end this subsection by remarking that similar formulae can be derived if $\xi$ is not necessarily positive. However, the system of Wiener-Hopf relations then becomes larger and no explicit results can be obtained unless some structure is imposed, for instance that $Z$ has downward phase-type jumps.

\section{Asymptotics of the maximum}

In this section, we study the tail asymptotics of $\mathrm{P}\left(\bar{Z}_{\infty}>x\right)$ and its local version, $\mathrm{P}\left(\bar{Z}_{\infty} \in\right.$ $(x, x+T])$, for fixed $T>0$ as $x \rightarrow \infty$, where $Z$ is a Lévy process drifting to $-\infty$. The motivation for studying this problem stems from risk theory; the probability $\mathrm{P}\left(\bar{Z}_{\infty}>x\right)$ is often called the ruin probability.

It is our aim to show that the embedding approach is a natural and powerful method for studying tail asymptotics for the maximum, regardless of the specific assumptions on the Lévy measure (e.g. that it be light tailed or heavy tailed). Relying on random walk results, for which we refer the reader to [25] for an overview, we study both the global and the local tail asymptotics in three different regimes. These regimes are respectively referred to as the Cramér case, the intermediate case, and the subexponential case. Interestingly, all known results on these asymptotics for Lévy processes have been derived on a case-by-case basis (indeed, the Cramér and global subexponential cases have been successfully examined elsewhere). Most importantly, since the embedding technique is a uniform approach that does not rely on the specific form of the tail of the Lévy measure, it allows us to fill the gaps in the literature. For instance, our results concerning the intermediate case and the local subexponential case are new. More results (and references) on asymptotics for Lévy processes can be found in [17] and [24].

In order to apply the embedding approach, we write $Z$ as a sum of two independent processes, $X$ and $Y$. Process $X$ has small jumps $\left(\Pi_{X}([1, \infty))=0\right)$, and process $Y$ is a compound Poisson subordinator with jumps exceeding 1 . This decomposition has recently been used by Doney [16] and Pakes [29] in the context of asymptotics. We set $\lambda=\Pi_{Z}([1, \infty)) \in[0, \infty)$, and write $\xi$ for a generic jump of $Y$; its distribution function is given by $z \mapsto \Pi_{Z}([1, z]) / \lambda$. If $\lambda=0$ then we set $\xi=0$. The random walk $\left\{S_{n}^{q}\right\}$ introduced in Subsection 2.1 plays an important role for $q=0$. For notational convenience, we write $S_{n}$ for $S_{n}^{0}$, i.e. $S$ is a random walk with step size distribution $\xi+X_{e_{\lambda}}$.

The process $X$ has a useful property: for any $\eta>0$, both $\operatorname{Eexp}\left(\eta \bar{X}_{e_{\lambda}}\right)$ and $\operatorname{Eexp}\left(\eta X_{e_{\lambda}}\right)$ are finite. Chernoff's inequality shows that both $\mathrm{P}\left(\bar{X}_{e_{\lambda}}>x\right)$ and $\mathrm{P}\left(X_{e_{\lambda}}>x\right)$ therefore decay 
faster than any exponential. To see that the moment generating functions are finite, first observe that, for $\operatorname{Re} \beta=0$, by the PRS identity,

$$
\operatorname{E} \exp \left(\beta X_{e_{\lambda}}\right)=\operatorname{E} \exp \left(\beta \bar{X}_{e_{\lambda}}\right) \operatorname{E} \exp \left(\beta \underline{X}_{e_{\lambda}}\right) .
$$

This identity can be extended to $\operatorname{Re} \beta>0$ by analytic continuation since, on this domain,

$$
\operatorname{Eexp}\left(\beta X_{e_{\lambda}}\right)=\frac{\lambda}{\lambda+\Psi_{X}(-\mathrm{i} \beta)}<\infty,
$$

where the finiteness follows from the fact that $\Pi_{X}$ is supported on $(-\infty, 1)$. It is trivial that $\operatorname{E} \exp \left(\beta \underline{X}_{e_{\lambda}}\right)$ is analytic for $\operatorname{Re} \beta>0$; hence, the claim is obtained.

\subsection{The Cramér case}

First we deal with the Cramér case, i.e. when there exists some $\omega \in(0, \infty)$ for which $\mathrm{Ee}^{\omega Z_{1}}=1$.

Given $\omega$, we can define an associated probability measure $\mathrm{P}^{\omega}$ such that $Z$ is a Lévy process under $\mathrm{P}^{\omega}$ with Lévy exponent $\Psi_{Z}(u-\mathrm{i} \omega)$. This measure plays an important role in the following result, which is due to Bertoin and Doney [8]. Even though the original proof is relatively short, it is instructive to see how the embedding approach recovers the result. The case where $Z$ has a discrete ladder structure is excluded, as random walk identities then directly apply.

Theorem 5. Let $Z$ be a Lévy process for which 0 is regular for $(0, \infty)$. Moreover, suppose that there is some $\omega \in(0, \infty)$ such that $\mathrm{E}^{\omega Z_{1}}=1$ and $\mathrm{E}_{1} \mathrm{e}^{\omega Z_{1}}<\infty$. Then, as $x \rightarrow \infty$ we have

$$
\mathrm{P}\left(\bar{Z}_{\infty}>x\right) \sim \frac{C_{\omega}}{\omega \mathrm{E} Z_{1} \mathrm{e}^{\omega Z_{1}}} \mathrm{e}^{-\omega x},
$$

where

$$
\log C_{\omega}=-\int_{0}^{\infty} t^{-1}\left(1-\mathrm{e}^{-t}\right)\left[\mathrm{P}\left(Z_{t}>0\right)+\mathrm{P}^{\omega}\left(Z_{t} \leq 0\right)\right] \mathrm{d} t .
$$

Moreover, for any $T>0$, as $x \rightarrow \infty$ we have

$$
\mathrm{P}\left(\bar{Z}_{\infty} \in(x, x+T]\right) \sim \frac{C_{\omega}}{\omega \mathrm{E} Z_{1} \mathrm{e}^{\omega Z_{1}}}\left(1-\mathrm{e}^{-\omega T}\right) \mathrm{e}^{-\omega x} .
$$

Proof. As the reader can readily verify, the second claim follows immediately from the first.

Let us study the random walk $S_{n}$ under the present assumptions. First note that $\mathrm{E} \mathrm{e}^{\omega Z_{1}}=1$ is equivalent to $\operatorname{Exp}\left(\omega X_{e_{\lambda}}\right) \mathrm{Ee}^{\omega \xi}=1$. Thus, by Lemma 1 of [21] (the step size distribution is nonlattice),

$$
\mathrm{P}\left(\sup _{n \geq 1} S_{n}>x\right) \sim \exp \left(-\sum_{n=1}^{\infty} \frac{1}{n}\left\{\mathrm{P}\left(S_{n}>0\right)+\mathrm{E}\left[\mathrm{e}^{\omega S_{n}} ; S_{n} \leq 0\right]\right\}\right) \frac{1}{\omega \mathrm{E} S_{1} \mathrm{e}^{\omega S_{1}}} \mathrm{e}^{-\omega x} .
$$

Since $\bar{X}_{e_{\lambda}}$ has a finite moment generating function, by Lemma 2.1 of [29] we have

$$
\begin{aligned}
\mathrm{P}\left(\bar{Z}_{\infty}>x\right) & =\mathrm{P}\left(\bar{X}_{e_{\lambda}}+\sup _{n \geq 1} S_{n}>x\right) \\
& \sim \exp \left(-\sum_{n=1}^{\infty} \frac{1}{n}\left\{\mathrm{P}\left(S_{n}>0\right)+\mathrm{E}\left[\mathrm{e}^{\omega S_{n}} ; S_{n} \leq 0\right]\right\}\right) \frac{\mathrm{E}^{\omega \bar{X}_{e_{\lambda}}}}{\omega \mathrm{E}_{1} \mathrm{e}^{\omega S_{1}}} \mathrm{e}^{-\omega x} .
\end{aligned}
$$


The rest of the proof consists of translating 'random walk terminology' into 'Lévy terminology'. For this, we suppose that the ladder process of $X$ is normalized such that, for $\alpha>0$ and $\beta \in \mathbb{R}$,

$$
\alpha+\Psi_{X}(\beta)=\kappa_{X}(\alpha,-\mathrm{i} \beta) \hat{\kappa}_{X}(\alpha, \mathrm{i} \beta)
$$

and similarly for $Z$.

The quantity $1-\mathrm{Ee}^{\mathrm{i} \beta S_{1}}=\Psi_{Z}(\beta) /\left(\lambda+\Psi_{X}(\beta)\right)$ has both a 'random walk' Wiener-Hopf decomposition and a 'Lévy' Wiener-Hopf decomposition, and their uniqueness leads to the identity

$$
\exp \left(-\sum_{n=1}^{\infty} \frac{1}{n} \mathrm{E}\left[\mathrm{e}^{\mathrm{i} \beta S_{n}} ; S_{n}>0\right]\right)=\frac{\kappa_{Z}(0,-\mathrm{i} \beta)}{\kappa_{X}(\lambda,-\mathrm{i} \beta)} .
$$

Similarly, since $1-\mathrm{Ee}^{(\omega+\mathrm{i} \beta) S_{1}}=\Psi_{Z}(\beta-\mathrm{i} \omega) /\left[\lambda+\Psi_{X}(\beta-\mathrm{i} \omega)\right]$, we have

$$
\exp \left(-\sum_{n=1}^{\infty} \frac{1}{n} \mathrm{E}\left[\mathrm{e}^{(\omega+\mathrm{i} \beta) S_{n}} ; S_{n} \leq 0\right]\right)=\frac{\hat{\kappa}_{Z}(0, \mathrm{i} \beta+\omega)}{\hat{\kappa}_{X}(\lambda, \mathrm{i} \beta+\omega)} .
$$

Using the facts that $\mathrm{E} \mathrm{e}^{\omega \bar{X}\left(e_{\lambda}\right)}=\kappa_{X}(\lambda, 0) / \kappa_{X}(\lambda,-\omega)$ (cf. Equation (VI.1) of [7]) and

$$
\mathrm{E} S_{1} \mathrm{e}^{\omega S_{1}}=\frac{\mathrm{E} Z_{1} \mathrm{e}^{\omega Z_{1}}}{\lambda E \mathrm{e}^{\omega \xi}}=\frac{\mathrm{E} Z_{1} \mathrm{e}^{\omega Z_{1}}}{\lambda+\Psi_{X}(-\mathrm{i} \omega)}=\frac{\mathrm{E} Z_{1} \mathrm{e}^{\omega Z_{1}}}{\kappa_{X}(\lambda,-\omega) \hat{\kappa}_{X}(\lambda, \omega)}
$$

(use $\mathrm{Ee}^{\omega Z(1)}=1$ ), the claim is obtained with $C_{\omega}=\kappa_{Z}(0,0) \hat{\kappa}_{Z}(0, \omega)$. Corollary VI.10 of [7] shows that $\log C_{\omega}$ is given by (5).

\subsection{The intermediate case}

This subsection studies the tail asymptotics of $\bar{Z}_{\infty}$ under the condition

$$
\delta=\sup \left\{\theta>0: \operatorname{Ee}^{\theta Z_{1}}<\infty\right\}>0,
$$

but we now suppose that we are in the intermediate case, i.e. that $\delta<\infty$ and $\mathrm{Ee}^{\delta Z_{1}}<1$. These assumptions imply that $\lambda \in(0, \infty)$.

If $D=1-\bar{D}$ is a probability distribution on $\mathbb{R}$, we write $D \in \delta(\alpha), \alpha>0$, if

1. $\lim _{x \rightarrow \infty} \bar{D}(x+y) / \bar{D}(x)=\mathrm{e}^{-\alpha y}$ for all $y \in \mathbb{R}$;

2. $\int_{-\infty}^{\infty} \mathrm{e}^{\alpha y} D(\mathrm{~d} y)<\infty$; and

3. $\lim _{x \rightarrow \infty} \overline{D^{(2)}}(x) / \bar{D}(x)=2 \int_{-\infty}^{\infty} \mathrm{e}^{\alpha y} D(\mathrm{~d} y)$,

where $D^{(2)}=D * D$ is the convolution of $D$ with itself. Note that the first requirement excludes the case where $D$ is concentrated on a lattice. More generally, if $\mu$ is a measure with $\mu([1, \infty))<\infty$, we write $\mu \in \delta(\alpha)$ if $\mu([1, \cdot]) / \mu([1, \infty)) \in \delta(\alpha)$.

We remark that if (6) holds and $\Pi_{Z} \in \delta(\alpha)$, then $\alpha$ necessarily equals $\delta$, as the reader can easily verify.

The following theorem builds on random walk results of [9] for step size distributions in $\delta(\alpha)$. It is closely related to Theorem 4.1 of [24], where the tail asymptotics were expressed in terms of characteristics of the ladder process. Using Proposition 5.3 of [24], this can be rewritten in terms of the characteristics of $Z$ itself (i.e. its Lévy measure). Here we find the asymptotics directly in terms of these characteristics (and the tail asymptotics for $Z_{1}$ ); this leads to a new asymptotic expression. Note that our underlying assumptions are the same as those of [24]. 
Theorem 6. Let $Z$ be a Lévy process drifting to $-\infty$ for which $\delta \in(0, \infty)$ and $\mathrm{E}^{\delta Z_{1}}<1$. If $\Pi_{Z} \in \delta(\delta)$ then $\mathrm{E}^{\delta \bar{Z}_{\infty}}<\infty$ and $\mathrm{P}\left(\bar{Z}_{\infty} \leq \cdot\right) \in \delta(\delta)$; in fact, as $x \rightarrow \infty$ we have

$$
\mathrm{P}\left(\bar{Z}_{\infty}>x\right) \sim-\frac{\mathrm{Ee}^{\delta \bar{Z}_{\infty}}}{\log \mathrm{Ee}^{\delta Z_{1}}} \Pi_{Z}((x, \infty)) \sim-\frac{\mathrm{Ee}^{\delta \bar{Z}_{\infty}}}{\mathrm{Ee}^{\delta Z_{1}} \log \mathrm{E} \mathrm{e}^{\delta Z_{1}}} \mathrm{P}\left(Z_{1}>x\right) .
$$

Moreover, under these assumptions, as $x \rightarrow \infty$ we have, for any $T>0$,

$\mathrm{P}\left(\bar{Z}_{\infty} \in(x, x+T]\right) \sim-\frac{\mathrm{Ee}^{\delta \bar{Z}_{\infty}}}{\log \mathrm{Ee}^{\delta Z_{1}}} \Pi_{Z}((x, x+T]) \sim-\frac{\mathrm{Ee}^{\delta \bar{Z}_{\infty}}}{\mathrm{Ee}^{\delta Z_{1}} \log \mathrm{Ee}^{\delta Z_{1}}} \mathrm{P}\left(Z_{1} \in(x, x+T]\right)$.

Proof. It suffices to prove the first asymptotic equivalences; for the proof of the relationship between the tail of the Lévy measure and the tail of the marginal distribution, we refer the reader to Theorem 3.1 of [29].

With the embedding in mind, we first note that, by Lemma 2.1 of [29], we have

$$
\mathrm{P}\left(\xi+X_{e_{\lambda}}>x\right) \sim \mathrm{E} \exp \left(\delta X_{e_{\lambda}}\right) \mathrm{P}(\xi>x) .
$$

Since $\mathrm{Ee}^{\delta Z_{1}}<1$ is equivalent to $\mathrm{Ee}^{\delta S_{1}}<1$, we may apply Theorem 1 of [9], which states (using Spitzer's identity) that

$$
\mathrm{P}\left(\sup _{n \geq 1} S_{n}>x\right) \sim \frac{\mathrm{E} \exp \left(\delta X_{e_{\lambda}}\right)}{1-\mathrm{E}^{\delta \xi} \mathrm{E} \exp \left(\delta X_{e_{\lambda}}\right)} \mathrm{E} \exp \left(\delta \sup _{n \geq 1} S_{n}\right) \mathrm{P}(\xi>x) .
$$

Some elementary calculations show that

$$
\frac{\mathrm{E} \exp \left(\delta X_{e_{\lambda}}\right)}{1-\mathrm{Ee}^{\delta \xi} \mathrm{E} \exp \left(\delta X_{e_{\lambda}}\right)}=\frac{1}{\left(\lambda+\Psi_{X}(-\mathrm{i} \delta) / \lambda\right)-\mathrm{Ee}^{\delta \xi}}=\frac{\lambda}{\Psi_{Z}(-\mathrm{i} \delta)}=-\frac{\lambda}{\log \mathrm{Ee}^{\delta Z_{1}}} .
$$

Using the fact that the moment generating function of $\bar{X}_{e_{\lambda}}$ is finite, we can again apply Lemma 2.1 of [29], to see that

$$
\begin{aligned}
\mathrm{P}\left(\bar{X}_{e_{\lambda}}+\sup _{n \geq 1} S_{n}>x\right) & \sim-\frac{\lambda}{\log \mathrm{E} \mathrm{e}^{\delta Z_{1}}} \mathrm{E} \exp \left(\delta \bar{X}_{e_{\lambda}}\right) \mathrm{E} \exp \left(\delta \sup _{n \geq 1} S_{n}\right) \mathrm{P}(\xi>x) \\
& =-\frac{\lambda \mathrm{Ee}^{\delta \bar{Z}_{\infty}}}{\log \mathrm{Ee}^{\delta Z_{1}}} \mathrm{P}(\xi>x)
\end{aligned}
$$

as claimed.

The second assertion is a consequence of the first claim and the observations that

$$
\mathrm{P}\left(\bar{Z}_{\infty}>x+T\right) \sim \mathrm{e}^{-\gamma T} \mathrm{P}\left(\bar{Z}_{\infty}>x\right) \text { and } \Pi_{Z}((x+T, \infty)) \sim \mathrm{e}^{-\gamma T} \Pi_{Z}((x, \infty)) .
$$

This completes the proof.

It is readily checked that the statements of this theorem are equivalent to

$$
\mathrm{P}\left(\bar{Z}_{\infty}>x\right) \sim-\frac{\delta \mathrm{Ee}^{\delta \bar{Z}_{\infty}}}{\log \mathrm{Ee}^{\delta Z_{1}}} \int_{x}^{\infty} \Pi_{Z}((y, \infty)) \mathrm{d} y .
$$

In this expression, we can formally let $\delta \rightarrow 0$, so that the prefactor of the integral tends to $1 / \mathrm{E} Z_{1}$. This naturally leads to the subexponential case. 


\subsection{The subexponential case}

A distribution function $D$ on $\mathbb{R}_{+}$is called subexponential, abbreviated as $D \in \delta$, if, in the notation of the previous subsection, $\overline{D^{(2)}}(x) \sim 2 \bar{D}(x)$. An important subclass of subexponential distributions have finite mean and satisfy

$$
\int_{0}^{x} \bar{D}(y) \bar{D}(x-y) \mathrm{d} y \sim 2 \int_{0}^{\infty} \bar{D}(y) \mathrm{d} y \bar{D}(x) ;
$$

we then write $D \in 8^{*}$. More generally, for a measure $\mu$, we write $\mu \in \delta$ or $\mu \in \delta^{*}$ if $\mu([1, \infty))<\infty$ and $\mu([1, \cdot]) / \mu([1, \infty)) \in \delta$ or, respectively, $\mu([1, \cdot]) / \mu([1, \infty]) \in \delta^{*}$.

Suppose that the integrated tail of the Lévy measure,

$$
\Pi_{I}((x, \infty))=\int_{x}^{\infty} \Pi_{Z}((y, \infty)) \mathrm{d} y
$$

is subexponential, i.e. $\Pi_{I} \in \delta$. It is known that this property is implied by $\Pi \in \varsigma^{*}$. Recall that we are interested in both the local and global tail asymptotics of $\bar{Z}_{\infty}$. As opposed to in the Cramér and intermediate cases, here the local asymptotics does not follow immediately from the global asymptotics. This local asymptotics, of which a proof is therefore nontrivial, appear here for the first time. Several different proofs have been given for the global version. For the first proof, we refer the reader to [3, Corollary 2.5]; see also [27], [12], [24], and [11].

Theorem 7. Let $Z$ be an integrable Lévy process with $\mathrm{E} Z_{1}<0$ and $\Pi_{I} \in$ s. Then

$$
\mathrm{P}\left(\bar{Z}_{\infty} \leq \cdot\right) \in \&
$$

in fact, as $x \rightarrow \infty$ we have

$$
\mathrm{P}\left(\bar{Z}_{\infty}>x\right) \sim-\frac{\int_{x}^{\infty} \Pi_{Z}((y, \infty)) \mathrm{d} y}{\mathrm{E} Z_{1}} \sim-\frac{\int_{x}^{\infty} \mathrm{P}\left(Z_{1}>y\right) \mathrm{d} y}{\mathrm{E} Z_{1}} .
$$

Moreover, if $\Pi_{Z} \in 8^{*}$ and $\Pi_{Z}$ is (ultimately) nonlattice, then as $x \rightarrow \infty$ we have, for any $T>0$,

$$
\mathrm{P}\left(\bar{Z}_{\infty} \in(x, x+T]\right) \sim-\frac{\int_{x}^{x+T} \Pi_{Z}((y, \infty)) \mathrm{d} y}{\mathrm{E} Z_{1}} \sim-\frac{\int_{x}^{x+T} \mathrm{P}\left(Z_{1}>y\right) \mathrm{d} y}{\mathrm{E} Z_{1}} .
$$

Proof. We have $\Pi_{Z}((x, \infty)) \sim \mathrm{P}\left(Z_{1}>x\right)$ (see, e.g. [29]); hence, it suffices to prove only the first equivalences.

Since $\Pi_{I} \in \mathcal{S}$, it is in particular long tailed, so, for $z \in \mathbb{R}$,

$$
\int_{x}^{\infty} \mathrm{P}(\xi>y+z) \mathrm{d} y \sim \int_{x}^{\infty} \mathrm{P}(\xi>y) \mathrm{d} y .
$$

Fix some $\eta>0$. The latter observation implies that the function $x \mapsto x^{\eta} \int_{1 \vee \log x}^{\infty} \mathrm{P}(\xi>y) \mathrm{d} y$ is locally bounded and regularly varying at infinity with index $\eta$, so that, by the uniform convergence theorem for regularly varying functions, for large $x$ we have

$$
\int_{x}^{\infty} \mathrm{P}(\xi>y-z) \mathrm{d} y \leq\left(1+\mathrm{e}^{\eta z}\right) \int_{x}^{\infty} \mathrm{P}(\xi>y) \mathrm{d} y,
$$


uniformly for $z \in[0, x-1]$. Since $X_{e_{\lambda}} \leq \bar{X}_{e_{\lambda}}$ and $\operatorname{Eexp}\left(\eta \bar{X}_{e_{\lambda}}\right)<\infty$, this implies that

$$
\begin{aligned}
\int_{x}^{\infty} \mathrm{P}(\xi+ & \left.X_{e_{\lambda}}>y\right) \mathrm{d} y \\
\leq & \int_{x}^{\infty} \mathrm{P}\left(\xi+\bar{X}_{e_{\lambda}}>y\right) \mathrm{d} y \\
= & O\left(\int_{x}^{\infty} \mathrm{P}(\xi>y) \mathrm{d} y\right)+\int_{x}^{\infty} \int_{(x-1, y-1]} \mathrm{P}(\xi>y-z) \mathrm{P}_{\bar{X}_{e_{\lambda}}}(\mathrm{d} z) \mathrm{d} y \\
& +\int_{x}^{\infty} \mathrm{P}\left(\bar{X}_{e_{\lambda}}>y-1\right) \mathrm{d} y
\end{aligned}
$$

and the last two terms are readily seen to be $O\left(\mathrm{P}\left(\bar{X}_{e_{\lambda}}>x\right)\right)$ and $O\left(\mathrm{e}^{-\eta x}\right)$, respectively. Using Chernoff's inequality and the fact that $\xi$ is heavy tailed, it follows that

$$
\int_{x}^{\infty} \mathrm{P}\left(\xi+X_{e_{\lambda}}>y\right) \mathrm{d} y=O\left(\int_{x}^{\infty} \mathrm{P}(\xi>y) \mathrm{d} y\right) .
$$

This shows that we can apply dominated convergence after Veraverbeke's theorem (see, e.g. [4, Theorem X.9.1(a)]), to see that

$$
\begin{aligned}
\mathrm{P}\left(\sup _{n \geq 1} S_{n}>x\right) & \sim-\frac{1}{\mathrm{E}\left[X_{e_{\lambda}}+\xi\right]} \int_{x}^{\infty} \mathrm{P}\left(X_{e_{\lambda}}+\xi>y\right) \mathrm{d} y \\
& \sim-\frac{1}{\mathrm{E}\left[X_{e_{\lambda}}+\xi\right]} \int_{x}^{\infty} \mathrm{P}(\xi>y) \mathrm{d} y .
\end{aligned}
$$

The right-hand side is readily seen to be equivalent to $\int_{x}^{\infty} \Pi_{Z}((y, \infty)) \mathrm{d} y /\left|\mathrm{E} Z_{1}\right|$, by definition of $\xi$. Since this is the tail of a subexponential random variable, the first claim follows from the fact that $\bar{X}_{e_{\lambda}}$ has a lighter tail.

The second assertion is proven similarly, but with Veraverbeke's theorem replaced by its local counterpart; see Equation (18) of [6]. The rest of the argument is simpler than that for the 'global' version, since $\mathrm{P}\left(X_{e_{\lambda}}+\xi>x\right) \sim \mathrm{P}(\xi>x)$ as $\Pi \in \mathcal{s}^{*} \subset$ \&. A lattice version can also be given. This proves the theorem.

A different proof for the first claim can be given based on recent results of Foss and Zachary [18]. Indeed, as noted in Section 4, a discrete-time Markov additive process is embedded in the lower diagram of Figure 1. In order to verify the assumptions of [18], we suppose that $Z$ is not spectrally positive, so that there exist $M_{-} \leq 0$ and $M_{+} \geq 0$ such that $\lambda_{ \pm}=\Pi_{Z}\left(\mathbb{R} \backslash\left(M_{-}, M_{+}\right)\right)<\infty$ and $\int_{\mathbb{R} \backslash\left(M_{-}, M_{+}\right)} z \Pi_{Z}(\mathrm{~d} z)<0$. We can write $\bar{Z}$ as a sum of $X$ and $Y$, where $Y$ is now a compound Poisson process with Lévy measure $\Pi_{Z}$ restricted to $\mathbb{R} \backslash\left(M_{-}, M_{+}\right)$. Further details are left to the reader.

\section{Acknowledgements}

The author thanks A. Kyprianou and D. Korshunov for helpful discussions, and M. Mandjes for carefully reading the manuscript.

The author is supported by the Netherlands Organization for Scientific Research (NWO) under grant 631.000.002. Part of this work was carried out during a visit to Université Paris VI, for which he wishes to acknowledge the DYNSTOCH network. He also received support from Science Foundation Ireland, grant number SFI04/RP1/I512. 


\section{References}

[1] Arjas, E. And Speed, T. P. (1973). A note on the second factorization identity of A. A. Borovkov. Theory Prob. Appl. 18, 576-578.

[2] Asmussen, S. (1995). Stationary distributions for fluid flow models with or without Brownian noise. Stoch. Models 11, 21-49.

[3] Asmussen, S. (1998). Subexponential asymptotics for stochastic processes: extremal behavior, stationary distributions and first passage probabilities. Ann. Appl. Prob. 8, 354-374.

[4] Asmussen, S. (2003). Applied Probability and Queues, 2nd edn. Springer, New York.

[5] Asmussen, S., Avram, F. And Pistorius, M. (2004). Russian and American put options under exponential phase-type Lévy models. Stoch. Process. Appl. 109, 79-111.

[6] Asmussen, S., Foss, S. And Korshunov, D. (2003). Asymptotics for sums of random variables with local subexponential behaviour. J. Theoret. Prob. 16, 489-518.

[7] Bertoin, J. (1996). Lévy Processes. Cambridge University Press.

[8] Bertoin, J. and Doney, R. A. (1994). Cramér's estimate for Lévy processes. Statist. Prob. Lett. 21, 363 -365.

[9] Bertoin, J. And Doney, R. A. (1996). Some asymptotic results for transient random walks. Adv. Appl. Prob. 28, 207-226.

[10] Boucherie, R. J., Boxma, O. J. And Sigman, K. (1997). A note on negative customers, GI/G/1 workload, and risk processes. Prob. Eng. Inf. Sci. 11, 305-311.

[11] Braverman, M., Mikosch, T. and Samorodnitsky, G. (2002). Tail probabilities of subadditive functionals of Lévy processes. Ann. Appl. Prob. 12, 69-100.

[12] Chan, T. (2004). Some applications of Lévy processes in insurance and finance. Finance (Revue Assoc. Française Finance) 25, 71-94.

[13] Choudhury, G. (2003). Some aspects of an M/G/1 queueing system with optional second service. Top 11, $141-150$.

[14] Dȩbicki, K., Dieker, A. B. ANd Rolski, T. (2006). Quasi-product forms for Lévy-driven fluid networks. To appear in Math. Operat. Res.

[15] Doney, R. A. (1987). On Wiener-Hopf factorisation and the distribution of extrema for certain stable processes. Ann. Prob. 15, 1352-1362.

[16] Doney, R. A. (2004). Stochastic bounds for Lévy processes. Ann. Prob. 32, 1545-1552.

[17] Doney, R. A. and Kyprianou, A. E. (2006). Overshoots and undershoots of Lévy processes. Ann. Appl. Prob. 16, 91-106.

[18] Foss, S. AND Zachary, S. (2002). Asymptotics for the maximum of a modulated random walk with heavytailed increments. In Analytic Methods in Applied Probability, American Mathematical Society, Providence, RI, pp. 37-52.

[19] Furrer, H. J. (1998). Risk processes perturbed by $\alpha$-stable Lévy motion. Scand. Actuarial J. 1998, 59-74.

[20] Huzak, M., Perman, M., ŠIKIĆ, H. AND VondračEK, Z. (2004). Ruin probabilities and decompositions for general perturbed risk processes. Ann. Appl. Prob. 14, 1378-1397.

[21] Iglehart, D. (1972). Extreme values in the GI/G/1 queue. Ann. Math. Statist. 43, 627-635.

[22] Jacod, J. And Shiryaev, A. N. (2003). Limit Theorems for Stochastic Processes, 2nd edn. Springer, Berlin.

[23] Kennedy, J. (1992). A probabilistic view of some algebraic results in Wiener-Hopf theory for symmetrizable Markov chains. In Stochastics and Quantum Mechanics, World Scientific, River Edge, NJ, pp. 165-177.

[24] Klüppelberg, C., Kyprianou, A. E. and Maller, R. A. (2004). Ruin probabilities and overshoots for general Lévy insurance risk processes. Ann. Appl. Prob. 14, 1766-1801.

[25] Korshunov, D. (1997). On distribution tail of the maximum of a random walk. Stoch. Process. Appl. 72, 97-103.

[26] Kou, S. And Wang, H. (2003). First passage times of a jump diffusion process. Adv. Appl. Prob. 35, 504-531.

[27] Maulik, K. and Zwart, B. (2006). Tail asymptotics for exponential functionals of Lévy processes. Stoch. Process. Appl. 116, 156-177.

[28] Mordecki, E. (2002). The distribution of the maximum of a Lévy process with positive jumps of phase-type. Theory Stoch. Process. 8, 309-316.

[29] Pakes, A. G. (2004). Convolution equivalence and infinite divisibility. J. Appl. Prob. 41, 407-424.

[30] Pistorius, M. (2006). On maxima and ladder processes for a dense class of Lévy process. J. Appl. Prob. 43, 208-220.

[31] Prabhu, N. U. (1998). Stochastic Storage Processes. Springer, New York.

[32] Rolski, T., Schmidli, H., Schmidt, V. and Teugels, J. L. (1999). Stochastic Processes for Insurance and Finance. John Wiley, Chichester.

[33] Schmidi, H. (2001). Distribution of the first ladder height of a stationary risk process perturbed by $\alpha$-stable Lévy motion. Insurance Math. Econom. 28, 13-20. 